Aus der Abteilung Kieferorthopädie

(Prof. Dr. med. dent. D. Kubein-Meesenburg)

im Zentrum Zahn-, Mund- und Kieferheilkunde

der Medizinischen Fakultät der Universität Göttingen

\title{
In-vitro-Untersuchung zur Prozessoptimierung bei Herstellung und adhäsiver Befestigung von 3-3-Retainern mit Hilfe einer Neodym-Magnetkette
}

\author{
Inaugural-Dissertation \\ zur Erlangung des Doktorgrades für Zahnheilkunde \\ der Medizinischen Fakultät \\ der Georg-August-Universität zu Göttingen
}

vorgelegt von

Wiebke Wasser-Merkel

aus

Marburg 
Dekan:

I. Berichterstatter:

II. Berichterstatter/in:

III. Berichterstatter/in:

Tag der mündlichen Prüfung:
Prof. Dr. Heyo K. Kroemer

PD Dr. med. dent. W. Hahn

Prof. Dr. med. dent. M. Hülsmann 


\section{Inhaltsverzeichnis}

1. Einleitung 5

2. Literaturübersicht

2.1 Retention 7

2.1.1 Kieferorthopädische Retention $\quad 7$

2.1.2 Retentionsgeräte 8

2.2 Der geklebte Retainer 9

2.2.1 Herstellung und Befestigung von 3-3-Retainern 12

$\begin{array}{ll}\text { 2.3 Magnete in der Zahnheilkunde } & 15\end{array}$

2.3.1 Grundlagen zu Magneten 15

2.3.2 Magnete und ihre Verwendung in der kieferorthopädischen Retention 16

$\begin{array}{ll}2.4 \text { Fragestellung } & 17\end{array}$

3. Material und Methoden $\quad 18$

3.1 Modellauswahl und Einteilung in Gruppen $\quad 18$

3.1.1 Archivierung und Datenschutz 19

3.2 Modellherstellung 20

$\begin{array}{ll}3.3 \text { Retainer } & 20\end{array}$

3.3.1 Drähte 20

3.3.2 Magnetkette 21

3.3.3 Herstellung der Retainer 22

3.3.3.1 Herstellung eines Retainers mit Einsatzhilfe 22

3.3.3.2 Herstellung eines Retainers mit Magnetkette 23

3.4 Adhäsive Befestigung 24

3.4.1 Adhäsive Befestigung eines Retainers mit Einsatzhilfe 24

3.4.2 Adhäsive Befestigung eines Retainers mit Magnetkette 25

3.5 Weiterbearbeitung der Modelle 25

3.5.1 Auswertung der Bilddateien $\quad 26$

$\begin{array}{ll}3.6 \text { Statistik } & 28\end{array}$

4. Ergebnisse 29

4.1 Auswertung der Fläche 29

4.2 Auswertung des senkrechten Abstandes $\quad 30$

4.3 Auswertung der Biegezeit 35

$\begin{array}{ll}\text { 4.4 Auswertung der Befestigungszeit } & 37\end{array}$ 
5. Diskussion $\quad 39$

5.1. Schlussfolgerung 44

6. Zusammenfassung 46

7. Literaturverzeichnis $\quad 48$

8. Anhang 57

8.1 Arbeitsanweisung für Zeitmess- und Passgenauigkeitsstudie 3-3-Retainer 57

8.2 Arbeitsanweisung für die Herstellung der 3-3-Retainer durch den

Zahntechniker $\quad 59$

8.3 Arbeitsanweisung für das Ausmessen der Bilddateien 61 


\section{$1 \quad$ Einleitung}

Nach Abschluss einer kieferorthopädischen Therapie mit aktiver Zahnbewegung kommt es häufig zu einer gewissen Rückstellung der Zähne in ihre Ausgangslage. Dieser Vorgang wird als Rezidiv bezeichnet und wird häufig in der Unterkieferfront beobachtet. Als eine Hauptursache der Rückstellung der Zähne ist der langsame Umbauprozess der Oxytalanfasern im suprakrestalen Bereich des Zahnhalteapparates nach aktiver Zahnbewegung zu sehen. Um ein Rezidiv zu vermeiden, sollte nach kieferorthopädischer Zahnbewegung eine Retentionsphase angeschlossen werden (Reitan 1969, Riedel 1960). Dabei wird mit herausnehmbaren oder festsitzenden Apparaturen versucht, die unerwünschte Rückstellung der Zähne zu vermeiden. Art und Dauer der Stabilisierung des Behandlungszieles sind abhängig von bestimmten Voraussetzungen des Patienten, die im Faserapparat zu suchen sind (Cronau et al. 2006). So zeigen Patienten mit Engstandsproblematik eine nach mesial gerichtete Vorspannung des Faserapparates, welche als Folge den Zahnbogen zusammenzieht (Cronau et al. 2006). Little et al. (1988), Little et al. (1981) und Schütz-Fransson et al. (1998) kamen zu dem Ergebnis, dass sich eine lange Retentionszeit gerade bei der Engstandsproblematik im Unterkiefer positiv auswirkt.

Ein sogenannter 3-3-Retainer ist ein festsitzender, hinter die Unterkieferfrontzähne geklebter Draht. Dieser passiv anliegende Draht soll helfen, ein Rezidiv zu vermeiden. Vorteil eines solchen festsitzenden Retainers ist eine dauerhafte Retention, die unabhängig von der Mitarbeit des Patienten ist (Heier et al. 1997). Misserfolge, die zum Verlust des Retainers führen, sind mehrheitlich im Befestigungsprozess zu suchen (Lumsden et al. 1999, Segner und Heinrici 2000). So gelten Spannungsfreiheit, korrekte Positionierung und Passivität während des Befestigungsvorganges als Grundvoraussetzung, um einen Bruch der Klebestelle, unerwünschte Zahnbewegung und somit auch einen Retentionsverlust zu vermeiden (Radlanski und Zain 2004).

In der Praxis ist seit Jahren ein Befestigungsprozess etabliert, bei dem mit Hilfe einer individuell gefertigten Einsatzhilfe der Retainer auf dem Zahnbogen platziert wird. Die Einsatzhilfe muss in einem eigenen Arbeitsgang durch einen Zahntechniker angefertigt werden und behindert oft den Klebevorgang am Patienten. Bei einer neu entwickelten Fixiertechnik mit einer Neodym-Magnet-Kette soll dem Behandler eine größere Übersicht beim Klebevorgang und eine zeitsparendere Behandlung ermöglicht werden.

Im Rahmen dieser Studie sollten das konventionelle und das magnetische Positionierungsverfahren für die adhäsive Befestigung der Drähte miteinander verglichen werden. Als Vergleichsparameter dienten die Dauer des Herstellungsprozesses eines 
Retainers durch einen Zahntechniker, die Dauer des adhäsiven Befestigungsvorganges durch einen Kieferorthopäden sowie die Passgenauigkeit der geklebten Retainer. Weiterhin sollte untersucht werden, ob unterschiedliche Drahttypen einen Einfluss auf die Dauer der Herstellungs- und Befestigungsprozesse sowie auf die Passgenauigkeit der Retainer haben. 


\section{$2 \quad$ Literaturübersicht}

\subsection{Retention}

\subsubsection{Kieferorthopädische Retention}

Die Retention nach kieferorthopädischer Behandlung wurde von Moyers (1973) als das notwendige Halten von Zähnen für einen gewissen Zeitraum in der Behandlungsendposition zum Erhalt des erzielten Ergebnisses beschrieben.

Es ist seit langem bekannt, dass nach kieferorthopädischer Zahnbewegung in den meisten Fällen eine Retentionsphase anzuschließen ist (Reitan 1969, Riedel 1960). Die Problematik nach abgeschlossener aktiver kieferorthopädischer Therapie liegt darin, dass Zähne, die durch Knochen bewegt wurden, die Tendenz zeigen, in ihre alte Position zurückzukehren (Bearn 1995). Die Form des Zahnbogens, vor allem im Unterkiefer, kann durch kieferorthopädische Apparaturen nicht dauerhaft verändert werden und der Knochen sowie das angrenzende Gewebe benötigen nach Abschluss der Behandlung einen gewissen Zeitraum, um sich reorganisieren zu können (Riedel 1960).

Die Gründe für ein Rezidiv sind jedoch weiterhin noch nicht vollkommen verstanden (Melrose und Millett 1998). Dem dentogingivalen Faserapparat wird bei der Rezidivproblematik eine entscheidende Rolle zugesprochen (Cronau et al. 2006, Ihlow et al. 2003). Reitan (1967) zeigte in seiner Studie, dass der Umbauprozess des dentogingivalen Faserapparates mindestens 232 Tage benötigt, um sich an die neue Position nach aktiver Zahnbewegung zu adaptieren. Weitere Faktoren, die zur Entstehung eines Rezidives führen können, sind Lippen-, Wangen- und Zungendruck, die Position der Zähne zueinander und das allgemeine körperliche Wachstum (Melrose und Millett 1998). Veränderungen in der Länge und Weite des Zahnbogens können bis in das vierte Lebensjahrzehnt und später auftreten. Dies ist ein physiologischer Prozess, der bei Patienten mit und ohne kieferorthopädische Therapie auftreten kann (Little et al. 1988, Little 1990).

Für die nach der aktiven kieferorthopädischen Therapie anschließende Retentionsphase gibt es kein einheitliches System, nach dem sich Behandler und Patient richten sollen, sodass es dem behandelnden Kieferorthopäden je nach Patientenfall überlassen wird, wie die Retentionsphase gestaltet wird (Zachrisson 1986).

Jedoch sollten nach Kahl-Nieke (2002) folgende Grundregeln bei der Retention beachtet werden:

- Zähne, die bewegt wurden, sollten retiniert werden, da sie die Tendenz haben, sich in ihre alte Position zurückzubewegen. 
- Das Ausschalten der Ursachen für eine Dysgnathie (z.B. Habits) beugt dem Rezidiv vor.

- $\quad$ Überkorrektur hat sich bei vielen Anomalien (z.B. Tiefbiss, Rotationen und Klasse III) bewährt und sollte zur Sicherheit durchgeführt werden.

- Eine ausreichende Retentionsdauer für die Reorganisation von Hart- und Weichgewebe sollte eingehalten werden.

- $\quad$ Korrekturen, die während des Wachstums durchgeführt werden, zeigen weniger Rezidive als andere.

- Die Zahnbogenform und -breite, vor allem diejenige des Unterkiefers, sollte möglichst nicht verändert werden, da in dieser Art kieferorthopädisch veränderte Zahnbögen in Richtung ihrer ursprünglichen Form rezidivieren.

- $\quad$ Bezüglich der Frage, ob eine perfekte Okklusion generell eine erfolgreiche Langzeitstabilität gewährleistet, gibt es keine Übereinstimmung. Sie trägt aber zumindest dazu bei, die Zähne in ihrer korrigierten Position zu halten.

Der in Göttingen entwickelte Retentionskatalog, der eine Einteilung nach Risikogruppen enthält, bietet dem Behandler einen Leitfaden für die Retentionsphase und dient dem Patienten als Informations- und Aufklärungsbogen. Der Ausgangsbefund eines Patienten (Ausprägung des Engstandsproblems) bildet die Grundlage der Einteilung in eine entsprechende Risikogruppe. Mit Hilfe dieses abgeleiteten Rezidivrisikos werden jedem Patientenfall Maßnahmen zur Stabilisierung des Behandlungsendergebnisses, wie Art des Retentionsgerätes und Dauer der Retentionsphase, zugeordnet. Anhand entsprechender Abbildungen können dem Patienten die einzelnen Retentionsmaßnahmen und die zeitliche Reihenfolge erläutert werden (Ihlow et al. 2005).

Der Grad der Veränderung des erzielten Therapieergebnisses ist variabel und kann nicht vorhergesagt werden. Deshalb sollte sich wenn möglich eine unbegrenzte Retentionsphase nach kieferorthopädischer Therapie anschließen (Little et al. 1988, Kaplan 1988, Nanda und Nanda 1992, Schütz-Fransson et al. 1998).

\subsubsection{Retentionsgeräte}

Angle (1907) beschrieb die ersten an Bändern fixierten Retentionsgeräte. Einige Jahre später entwickelte Hawley (1919) eine herausnehmbare Plattenapparatur zur Retention im Oberkiefer. 
Seit Ende der 70er Jahre werden zunehmend geklebte 3-3-Retainer in der Kieferorthopädie zur Rezidivprophylaxe eines Unterkieferengstandes eingesetzt (Zachrisson 1977).

3-3-Retainer sind kleine, an die linguale Fläche der Zähne geklebte Drähte, die heute das Mittel der Wahl für eine ästhetische Langzeitretention darstellen (Segner und Heinrici 2000).

\subsection{Der geklebte Retainer}

Nach Einführung der Säure-Ätz-Technik (Buonocore 1955) in die Zahnheilkunde berichtete Newman (1965) darüber, orthodontische Apparaturen an die Zahnoberfläche direkt zu kleben. Knierim (1973) stellte erstmals eine Technik dar, mit der auf Basis der Säure-Ätz-Technik ein Draht mit Komposit an die lingualen Zahnflächen der unteren Eckzähne geklebt wurde.

In der Literatur werden verschiedensten Arten von geklebten Retainern beschrieben. Zachrisson (1977, 1995) beschrieb drei Generationen von 3-3-Unterkieferlingualretainern. Die Retainer der „ersten Generation“ bestanden aus einem einfachen, runden Blue-Elgiloy ${ }^{\circledR}$ Draht (0,032 inch - 0,036 inch) mit einer Schlaufe als Retention an jedem Ende. Anfang der achtziger Jahre wurden diese durch einen verseilten, flexiblen Draht (0,032 inch) ersetzt. Durch die Drahtspiralen wurde eine ausreichende Retention gewährleistet, sodass die Schlaufen überflüssig wurden und die Befestigung erleichtert wurde (Årtun und Zachrisson 1982). Als Nachteil dieser Retainer der „Zweiten Generation" wurde der Draht selbst gesehen. Trotz der Verwendung eines Drahtes mit dem größten zur Verfügung stehenden Durchmesser (0,032 inch), erschien er nicht stabil genug. In einigen Fällen kam es dazu, dass sich der Retainer löste und verformte (Zachrisson 1995). 1995 beschrieb Zachrisson den Retainer der „dritten Generation". Hierbei handelt es sich um einen glatten Edelstahldraht (0,030 inch 0,032 inch), der auf einem Hartgipsmodell den Lingualflächen der unteren Inzisivi angebogen wird. Eine erhöhte Retention des Komposits wird durch Sandstrahlen der Drahtenden erreicht. Neben diesen 2-Basen-Retainern, die nur an den Eckzähnen befestigt wurden, wurden auch Retainer aus flexiblen verseilten Drähten entwickelt, die an jedem Zahn des unteren FrontEckzahnsegments befestigt wurden, sogenannte 6-Basen-Retainer (Zachrisson 1977, 1982, 1985, 1986, Dahl und Zachrisson 1991). Der Vorteil neben der erhöhten mechanischen Retention (Zachrisson 1983) ist, dass dieser flexible Draht eine physiologische Zahnbeweglichkeit zulässt, auch wenn der Draht an benachbarten Zähnen fixiert ist (Årtun 1984, Watted et al. 2001).

Es wurden verschiedene Durchmesser der Drähte oder unterschiedliche Komposite beschrieben (Rubenstein 1976, Carter 1978, Lubit 1979, Lee RT 1981, Zachrisson 1977, Eade 1980). Außerdem wurden Retainer auf einer vorgefertigten Basis (Chen 1978, Weisser 1978), 
die Verwendung von Drahtnetzen in Kombination mit Komposit (Gazit und Leiberman 1976, Rosenberg 1980) sowie die Verwendung von Retainern aus Glasfaserstreifen (Diamond 1987, Orchin 1990, Moser und Moser 1996) oder Polyethylen-Bändern (Rose et al. 2002) dargestellt.

Gegenwärtig werden vor allem zwei Arten von 3-3-Retainern in der Praxis verwendet. Man unterscheidet 2-Basen- und 6-Basen-Retainer (Butler und Dowling 2005). Ein 3-3-Retainer aus starrem Draht, der nur an den Eckzähnen (2-Punkt-Retainer) befestigt wird, wie ihn Zachrisson (1995) beschreibt, wird als 2-Basen-Retainer bezeichnet. Er wird vor allem verwendet, um die interkanine Weite stabil zu halten. Bei Verwendung eines 2-BasenRetainers besteht allerdings die Gefahr eines Rezidivs, vor allem in Form von Rotationen und Protrusion der nicht in den Retainer eingebundenen Schneidezähne (Zachrisson 1986, Störmann und Ehmer 2002).

Ein 6-Basen-Retainer wird aus verseiltem, flexiblem Draht hergestellt und ist an jedem einzelnen Zahn des Front-Eckzahnsegments befestigt. Er soll zusätzlich Rotationen einzelner Zähne verhindern (Butler und Dowling 2005).

Retainer weisen unterschiedliche Nachteile wie zum Beispiel die Gefahr des Verlustes, negative Einflüsse auf das Parodont oder die mangelnde Hygienefähigkeit auf (Bearn 1995).

Der Verlust eines Retainers kann verschiedene Ursachen haben (Butler und Dowling 2005, Lee KD und Mills 2009). Ein Ablösen kann an der Verbundstelle zwischen Schmelz und Adhäsiv stattfinden (Butler und Dowling 2005, Bearn 1995). Diese Art des Verlustes kann auch als adhäsiver Bruch bezeichnet werden. Eine weitere Ursache für einen Retainerverlust kann ein Bruch des Verbundes zwischen Draht und Komposit sein (kohäsiver Bruch) (Radlanski und Zain 2004, Lee KD und Mills 2009). Die höchste Verlustrate eines Retainers wird durch ein Ablösen an der Schmelz-Komposit-Verbindung (adhäsiver Bruch) verursacht. Årtun und Zachrisson (1982) zeigten eine Ablöserate von 30,8 \% für einen 2-Basen-Retainer der Stärke 0,032 inch aus mehrfach verseiltem Draht in einem Untersuchungszeitraum von 3 Jahren. Die Verlustrate eines 2-Punkt-Retainers aus planem Draht der gleichen Stärke zeigte eine Verlustrate von 9,1\% bei gleichem Untersuchungszeitraum.

Studien über die Verlustrate von 6-Basen-Retainern aus mehrfach verseiltem Draht der Stärke 0,0215 inch zeigten eine Verlustrate von 5,9\% in einem Zeitraum von 3,2 Jahren (Dahl und Zachrisson 1991) oder eine Verlustrate von 0,55 \% pro Retainer und Jahr (Segner und Heinrici 2000).

Untersuchungen von Radlanski und Zain (2004, S. 334) zeigten, dass „der Retainerdraht möglichst nur geringe Hebelwirkungen erlauben sollte.“ Diese Hebelwirkungen verstärken 
die einwirkende Kraft auf die Verbundstelle zwischen Zahnoberfläche und Draht und verstärken somit die Bruchgefahr. Aus diesem Grund „sollte beim Anpassen des Drahtes darauf geachtet werden, dass frei gespannte Drahtstrecken als mögliche Angriffspunkte für Abzugskräfte möglichst kurz gehalten werden“ (Radlanski und Zain 2004, S. 334).

Da bei 6-Basen-Retainern die Abzugskräfte auf mehrere Klebestellen verteilt werden können und somit eine geringere Verlustrate $\mathrm{zu}$ erwarten ist, sollen diese 2-Basen-Retainern vorgezogen werden (Radlanski und Zain 2004).

Zudem besteht ein Zusammenhang zwischen dem Durchmesser und damit einer größeren Steifigkeit des Drahtes mit einer erhöhten Ablöserate (Radlanski und Zain 2004).

Dickere Drähte folgen der physiologischen Zahnbewegung schlechter als dünnere flexiblere Drähte, und daher sind die an den Klebestellen wirkenden Scherkräfte höher (Störmann und Ehmer 2002).

Ein häufig diskutierter Nachteil geklebter Retainer ist die Einschränkung der Zahnbeweglichkeit, "die auf Dauer zu einer Inaktivitätsatrophie führen könnte." (Schwarze et al. 1995, S. 26). Watted et al. (2001) zeigten, dass die Zahnbeweglichkeit durch die Anzahl der in den Lingualretainer einbezogenen Zähne bestimmt wird. Ein 2-Basen-Retainer lässt eine höhere physiologische Zahnbeweglichkeit zu als ein 6-Basen-Retainer. Aus diesem Grund erscheinen Retainer aus 0.015 inch verseiltem Draht für einen 6-Basen-Retainer aus parodontalphysiologischer Sicht empfehlenswert, da bei dieser Drahtstärke die größtmögliche Flexibilität gewährleistet ist (Schwarze et al. 1995). Es konnte jedoch in einer Studie von Andrén et al. 1998 über einen Zeitraum von im Durchschnitt 6,8 Jahren gezeigt werden, dass die Verlustrate eines Retainers aus 0,015 inch starkem mehrfach verseiltem Draht signifikant höher ist als die eines Retainers aus 0,0195 inch starkem mehrfach verseiltem Draht. So sollte die Indikation für Retainer der Drahtstärke 0,015 inch vor allem auf parodontale Risikofälle beschränkt werden (Zachrisson 1983), da sie durch die erhöhte Flexibilität die physiologische Zahnbeweglichkeit am wenigsten einschränken.

Die Hygienefähigkeit wird als ein Nachteil jeglicher Art geklebter Retainer gesehen (Butler und Dowling 2005). Dies ist dadurch zu erklären, dass die dauerhafte Abdeckung großer Anteile der lingualen Zahnoberflächen mit Befestigungskomposit und ein lingual angebrachter durchgehender Drahtbogen, die Anwendung von Zahnseide und anderen Pflegemitteln deutlich einschränkt. Ein geklebter Retainer scheint auch eine Prädilektionsstelle für die Ansammlung von Plaque und Zahnstein zu sein (Årtun 1984, Butler und Dowling 2005, Dahl und Zachrisson 1991, Heier et al. 1997). Trotz Aufklärung der Patienten über adäquate Mundhygiene zeigen Patienten mit geklebten Retainern von 
Kontrolltermin zu Kontrolltermin eine gesteigerte Plaqueakkumulation (Störmann und Ehmer 2002). Jedoch ist trotz erhöhter Plaque- und Zahnsteinansammlung entlang eines lingual geklebten Retainers keine erhöhte Kariesrate oder eine Zunahme der parodontalen Infektionen der angrenzenden Hart- und Weichgewebe festzustellen (Årtun et al. 1997, Dahl und Zachrisson 1991, Gorelick et al. 1982).

Es konnte in keiner Studie gezeigt werden, dass durch einen geklebten Retainer ein Nachteil für die Mundgesundheit entsteht (Bearn 1995).

Heute stellt der 6-Basen-Retainer aus einem mehrfach verseilten Draht das Mittel der Wahl für eine ästhetische Langzeitretention dar, denn er gilt als zuverlässig, unabhängig von der Mitarbeit des Patienten, relativ leicht herzustellen, ist nahezu unsichtbar und besitzt eine hohe Patientenakzeptanz (Axelsson und Zachrisson 1992, Bearn 1995, Dahl und Zachrisson 1991, Heier et al. 1997, Segner und Heinrici 2000, Störmann und Ehmer 2002, Zachrisson 1977, Zachrisson 1997).

Folgende Indikationen wurden für die Befestigung geklebter Retainer formuliert (Lee RT 1981, Watted et al. 2001, Zachrisson 1983):

- $\quad$ Grenzwertig labial gekippte Frontzähne

- $\quad$ Nach Auflösung ausgeprägter Frontengstände

- $\quad$ Bei knappen vertikalen Verhältnissen in der Front

- $\quad$ Prätherapeutisch große Lücken in der Front bzw. Diastema mediale

- $\quad$ Prätherapeutisch starke Rotationen

- $\quad$ Extraktion eines unteren Inzisivus

- $\quad$ Klasse-III-Patienten

- $\quad$ Retrudierte Fronten bei Deckbiss und neutraler Bisslage

- $\quad$ Erwachsenenbehandlung mit zu erwartender Zahnwanderung nach Behandlung.

\subsubsection{Herstellung und Befestigung von 3-3-Retainern}

Für die langfristige Überlebensrate des Retainers spielen neben dem Durchmesser des Drahtes vor allem exakte Herstellungs- und Befestigungstechniken eine große Rolle.

Man unterscheidet grundsätzlich direkt am Patienten hergestellte 6-Basen-Retainer von solchen, die nach Abdruck und Modellherstellung im Labor von einem Zahntechniker gefertigt werden (Bearn 1995). Bei der direkten Herstellung eines 3-3-Retainers wird ein Draht ohne Schlaufen an den Enden verwendet (Zachrisson 1983, Shah et al. 2005). Die Passung des Drahtes sowie sein passiver Sitz werden im Mund des Patienten kontrolliert 
(Bearn 1995). Bevor der Retainer geklebt wird, müssen die Zahnoberflächen sorgfältig gereinigt sowie der Schmelz angeätzt werden (Knierim 1973).

Um optimale Voraussetzungen für den adhäsiven Befestigungsvorgang zu schaffen, müssen verschiedene Kriterien berücksichtigt werden, da vor allem Fehler bei diesem Arbeitsschritt als Ursache für Misserfolge und Retentionsverlust zu sehen sind (Lumsden et al. 1999, Segner und Heinrici 2000).

Eine wichtige Rolle für die erfolgreiche adhäsive Befestigung des Retainers spielen die Geschwindigkeit des Klebeprozesses, die Übersichtlichkeit des Arbeitsfeldes sowie die passive und korrekte Positionierung des Retainers. Die Bedeutung der Arbeitsgeschwindigkeit ist dadurch $\mathrm{zu}$ erklären, dass mit zunehmender Dauer die Wahrscheinlichkeit einer Kontamination der geätzten Schmelzoberfläche mit Blut, Speichel oder Sulkusflüssigkeit zunehmend steigt. Außerdem wird während dieser Zeit kontinuierlich Feuchtigkeit aus der Atemluft auf die konditionierte Schmelzoberfläche gebracht. Alle diese Faktoren führen zu einer erhöhten Wahrscheinlichkeit für eine Reduktion der Festigkeit des adhäsiven Verbundes. Einer der häufigsten Fehler während des adhäsiven Befestigungsprozesses ist dabei die Kontamination der Zahnoberfläche mit Speichel. Dadurch wird der Verbund zwischen Zahn und Befestigungsmaterial primär instabil (Axelsson und Zachrisson 1992).

Ein übersichtliches Arbeitsfeld erleichtert dem Behandler den Klebevorgang, wodurch ein schnellerer Arbeitsvorgang gewährleistet wird. Dies wiederum reduziert die Wahrscheinlichkeit der Kontamination der geätzten Schmelzoberfläche, was letztlich die Überlebensrate des 3-3-Retainers beeinflusst (Zachrisson 1982, Dahl and Zachrisson 1991, Andrén et al. 1998, Hahn et al. 2008). Für die temporäre Fixierung des Retainers wurden zahlreiche Techniken entwickelt, die konstruktiv bedingt eine unterschiedlich ausgeprägte Übersicht des Arbeitsfeldes erlauben. Zachrisson (1983), Osborn (1983), Paulson (1992), Cohen et al. (1994), Cook (2002) und Hahn et al. (2008) beschreiben Techniken, bei denen mit Hilfe von Zahnseide der Draht fixiert wird. Bei dieser Technik wird Zahnseide in den Interdentalraum eingebracht, und der Draht wird mit Hilfe von lingual gebildeten Schlaufen gehalten. Durch ein Verrutschen der Zahnseide kann der Retainer in einer falschen Position befestigt werden. Außerdem kann es zu einer Irritation der Gingiva durch die Zahnseide kommen, wodurch sich das Risiko der Kontamination der Zahnoberfläche mit Blut oder Sulkusflüssigkeit während des Klebevorgangs erhöht (Hahn et al. 2008).

Ähnlich wie die Technik mit Zahnseide beschreiben andere Autoren die Fixation des Retainers mit Drahtligaturen (Årtun und Zachrisson 1982, Al-Emran und Barakati 2007) oder 
mit kieferorthopädischen Elastics (Carter 1978, Meyers und Vogel 1982, Read 1984, Shah et al. 2005).

Eade (1980) beschreibt eine Technik, bei der mit Hilfe von autopolymerisierendem Kunststoff Übertragungskappen am Retainer befestigt werden, welche an den Eckzähnen lokalisiert sind. Nach abgeschlossenem Klebevorgang werden diese Kappen entfernt.

Auch Hobson und Eastaugh (1993), Zekic und Gelgör (2004) und Acharya et al. (2004) beschreiben Methoden zur Erleichterung des Klebevorgangs mit Übertragungshilfen aus Silikon oder Kunststoff. Bei Techniken mit Übertragungshilfen, die fest mit dem Draht verbunden sind, besteht die Gefahr, dass bei Entfernung der Übertragungskappe zu viel Spannung auf den Draht ausgeübt wird und es so zu einem kohäsiven Bruch kommen kann. Ein Vorteil der Methoden mit vorgefertigten Übertragungshilfen ist eine gewisse Zeitersparnis am Patienten, da die Übertragungshilfe bereits im Labor gefertigt und mit dem Retainer verbunden bereitgestellt wird. Außerdem ist so auch die Position des Drahtes festgelegt, was die Zeit für die Bestimmung einer korrekten Lage des Retainers an der lingualen Zahnoberfläche deutlich verkürzt.

Eine weitere Methode zur Fixierung des Retainers während des Klebevorgangs ist die sogenannte Vier-Hand-Technik, bei der der Draht vom Behandler gehalten wird, während die Helferin ihn befestigt (Dahl und Zachrisson 1991). Vor allem bei dieser Methode ist die Übersichtlichkeit des Arbeitsfeldes beeinträchtigt.

Neben den oben beschriebenen Voraussetzungen für einen langfristigen Retainerhalt ist auch die Spannungsfreiheit der adhäsiven Befestigung von entscheidender Bedeutung. Dafür ist eine passive und korrekte Positionierung des Drahtes eine wichtige Voraussetzung (Störmann und Ehmer 2002). Studien von Årtun und Zachrisson (1982) und Zachrisson (1986) wiesen eine erhöhte Ablöserate der Retainer nach, wenn diese Voraussetzungen nicht eingehalten werden. Spannungsfreiheit ist Grundvoraussetzung dafür, dass es nicht zu einem Bruch an einer Klebestelle, zu unerwünschten Zahnbewegungen und damit zum Retentionsverlust kommt (Radlanski und Zain 2004).

Ein weiteres Problem bei der Befestigung des Retainers liegt in der korrekten Positionierung der Kunststoffbasen zur Befestigung des Drahtes (Zachrisson 1977, Årtun et al. 1997, Dahl und Zachrisson 1991).

Dahl und Zachrisson (1991) beschrieben eine Abrasion des Kunststoffs und eine erhöhte Anzahl adhäsiver Brüche durch mechanische Kräfte wie Zähneputzen und den Kauvorgang. Aus diesem Grund sollten okklusale Interferenzen vermieden und eine ausreichende Menge an Komposit zur Befestigung des Retainers verwendet werden. 


\subsection{Magnete in der Zahnheilkunde}

Magnete finden in der Zahnheilkunde vor allem zur Fixation von Prothesen und Teilprothesen seit Jahren klinische Anwendung (Javid 1971, Federick 1976, Gillings 1981). In der Kieferorthopädie werden Magnete zur Einordnung retinierter Zähne (Sandler 1991, Darendeliler und Friedle 1994), zur Expansion (Vardimon 1987), zur Retention (Springate und Sandler 1991), zur Korrektur des offenen Bisses (Dellinger 1986), zur Zahnbewegung entlang fixierter Bögen (Blechman 1985) und in funktionskieferorthopädischen Apparaturen (Vardimon et al. 1989, 1990, Darendeliler und Joho 1993, Darendeliler et al. 1993) verwendet.

Vorteil von Magneten in der Kieferorthopädie ist, dass sie eine anziehende oder abstoßende Wirkung haben können.

\subsubsection{Grundlagen zu Magneten}

Magnete erzeugen ein magnetisches Kraftfeld, dessen Feldlinien vom sogenannten Nordpol des Magneten zu seinem Südpol verlaufen (Noar und Evans 1999). Die Stärke des magnetischen Feldes, die man Magnetische Flussdichte (Einheit Tesla) nennt, beschreibt die Stärke der Wechselwirkung mit einem anderen in dem Feld befindlichen Magneten und bewirkt das Anziehen oder Abstoßen anderer magnetischer Materialien (Noar und Evans 1999).

„In ein magnetisches Feld eingebrachte Stoffe können die magnetische Wirkung schwächen oder verstärken“ (Harms 2000, S. 131). Dabei unterscheidet man zwischen diamagnetischen, paramagnetischen und ferromagnetischen Materialien. Diamagnetische Stoffe wie z. B. Gold, magnetisieren sich in einem Magnetfeld und verdrängen dabei die magnetischen Feldlinien in ihrem Inneren, sodass das magnetische Feld innerhalb des Stoffes schwächer ist als außerhalb. Paramagnetische Materialien, z. B. Aluminium, magnetisieren sich durch ein externes Magnetfeld, sodass sich das Magnetfeld in ihrem Inneren verstärkt. Paramagnetische Materialien sind jedoch nicht in der Lage diese Magnetisierung ohne das externe Feld aufrecht zu erhalten wie die Gruppe der Ferromagneten. Ferromagnetische Stoffe wie Eisen, Nickel und Kobalt können im Vergleich zu diamagnetischen oder paramagnetischen Stoffen ein magnetisches Feld um ein tausendfaches erhöhen, da sie die Eigenschaft der Remanenz besitzen. Das bedeutet, dass sie magnetisch bleiben, nachdem man sie aus dem Magnetfeld entfernt hat.

In den siebziger Jahren wurden Magnete entwickelt, die in der Lage sind, große Kräfte im Vergleich zu ihrer kleinen Größe zu entwickeln (Noar und Evans 1999). Grundlage dieser 
Eigenschaft ist die magnetokristalline Richtungsabhängigkeit dieser Magnete. Diese erlaubt einzelnen Kristallen, sich vorzugsweise entlang einer Richtung, ihrer C-Achse, anzulagern. Dadurch wird der Magnetismus verstärkt (Harris 1990). Magnete aus Samarium-Kobalt $\left(\mathrm{SmCo}_{5}\right)$ oder Neodym-Eisen-Bor $\left(\mathrm{Nd}_{2} \mathrm{Fe}_{14} \mathrm{~B}\right)$ besitzen diese Eigenschaft und haben außerdem eine hohe Resistenz gegen Entmagnetisierung (Noar und Evans 1999). Nachteile dieser Magnete sind ihre Sprödigkeit, ihre Korrosionsanfälligkeit (Wilson et al. 1995, Wilson et al. 1997) und der Verlust von magnetischer Kraft, wenn sie erhitzt werden (Noar und Evans 1999).

Es wurden verschiedenste Studien zur biologischen Sicherheit von Magneten in der Zahnheilkunde durchgeführt. Vardimon und Mueller (1985) veranschaulichten, dass Magnete, die Neodym enthalten, anfällig für Korrosion sind und somit potentiell toxische Stoffe freisetzen können. In anderen Studien konnte gezeigt werden, dass diese Korrosionsprodukte eine milde Zytotoxizität aufweisen (Sandler et al. 1989, Bondemark et al. 1994a, Bondemark et al. 1994b, Bondemark et al. 1994c). Werden neodymhaltige Magnete mit Parylene beschichtet, so entsteht ein effektiver Schutz vor Korrosion (Wilson et al. 1995). Bondemark et al. (1995) zeigten, dass das statische magnetische Feld, welches zum Beispiel durch $\mathrm{Nd}_{2} \mathrm{Fe}_{14} \mathrm{~B}$-Magnete entsteht, keine negativen Einflüsse auf die Pulpa oder das umliegende Weichgewebe hat.

$\mathrm{Nd}_{2} \mathrm{Fe}_{14} \mathrm{~B}$-Magnete sollten nicht bei Patienten mit Herzschrittmachern verwendet werden ( $\mathrm{Li}$ 2007, Wolber et al. 2007).

\subsubsection{Magnete und ihre Verwendung in der kieferorthopädischen Retention}

1991 beschrieben Springate und Sandler einen mikromagnetischen Retainer zur Retention der zentralen Inzisivi im Oberkiefer nach kieferorthopädischem Schluss eines Diastema mediale. Hierzu wurden nach aktiver kieferorthopädischer Therapie zwei kleine $\mathrm{Nd}_{2} \mathrm{Fe}_{14} \mathrm{~B}$-Magnete an der Palatinalfläche der Inzisivi mit Hilfe von Komposit befestigt. Vorteil dieser Technik ist, dass die Zähne nicht durch einen Draht geschient werden. Dadurch werden die Zähne in ihrer physiologischen Zahnbewegung nicht eingeschränkt und die Hygienefähigkeit bleibt gewährleistet. Durch den nur palatinal angebrachten kontinuierlichen horizontalen Zug kann es bei dieser Art von Retention jedoch auch nach aktiver Therapie noch zu unerwünschten Zahnbewegungen, wie z. B. Rotationen um die Längsachse der Zähne, kommen. Es ist auch als problematisch anzusehen, dass in Okklusion die Unterkieferinzisivi auf die Magnete beißen. So kann die Kunststoffschicht der Magnete beschädigt werden oder es kann zu einem Magnetverlust kommen. Als Folge könnte es zu einem direkten Kontakt der Magnete mit der 
Mundhöhle kommen und es bestünde die Gefahr der Beschädigung durch Korrosion (Noar und Evans 1999).

In einem von Hahn et al. (2008) veröffentlichten Artikel werden Magnete nicht zur dauerhaften Retention, also im Mund verbleibend, beschrieben, sondern lediglich als Hilfsmittel zur temporären Fixierung des Retainerdrahtes während der adhäsiven Befestigung. Hierzu haben die Autoren eine $\mathrm{Nd}_{2} \mathrm{Fe}_{14} \mathrm{~B}$-Magnetkette entwickelt. Der Retainer wird durch die $\mathrm{Nd}_{2} \mathrm{Fe}_{14} \mathrm{~B}$-Kette beim Klebevorgang in Position gehalten, sodass der Behandler beide Hände frei hat, um das Komposit aufzutragen und eine maximale Übersicht während des Klebevorgangs gewährleistet ist. In dieser Studie konnte gezeigt werden, dass durch diese Befestigungstechnik der klinische Befestigungsprozess zeitlich optimiert werden kann.

\subsection{Fragestellung}

Ziel der vorliegenden Studie war es, eine gebräuchliche konventionelle Befestigungsmethode und die Neodym-Magnetkette für die adhäsive Befestigung unterschiedlicher Drähte zu vergleichen.

Dabei sollte auf folgende Fragen eingegangen werden:

- Kann der Herstellungs- und Befestigungsvorgang von 3-3-Retainern mit Hilfe der Neodym-Magnetkette zeitlich optimiert werden?

- Ist die Passgenauigkeit eines mit Hilfe der Magnetkette geklebten 3-3-Retainers vergleichbar oder besser als die eines mit Hilfe einer vorgefertigten Übertragungshilfe geklebten Retainers?

- Hat die Drahtqualität Einfluss auf die Dauer des Herstellungs- und Klebevorgangs sowie auf die Passgenauigkeit des Retainers? 


\section{$3 \quad$ Material und Methoden}

In der vorliegenden Studie sollen zwei unterschiedliche Methoden zur temporären Fixation von lingual geklebten 3-3-Retainern verglichen werden. Bei den Methoden handelt es sich um eine konventionelle Methode mit einer laborgefertigten Einsatzhilfe und eine Methode mit einer neu entwickelten Neodym-Magnetkette. Als Parameter dienen die Geschwindigkeit der Herstellung und der Befestigung der Retainer. Als weiteres Maß dient die Passgenauigkeit der Retainer. Außerdem sollte der Einfluss des Drahttyps auf die Passung sowie auf die Dauer des Herstellungs- und Befestigungsvorgangs der Retainer untersucht werden.

Der Studienablauf wurde von der Ethikkommission der Georg-August-Universität überprüft und genehmigt (Antrag 8/1/07).

\subsection{Modellauswahl und Einteilung in Gruppen}

Zur Klärung der gestellten Fragen wurden an Modellen des Unterkiefers Drahtbogenretainer geklebt und anschließend vermessen. Hierfür wurden 20 Modelle abgeschlossener Behandlungsfälle aus dem Patientengut der Abteilung Kieferorthopädie der Universitätsmedizin Göttingen herausgesucht. Um auch einen Einfluss der Zahnstellung beurteilen zu können, wurden sowohl Modelle mit Engstand in der Unterkieferfront als auch solche mit geraden Zähnen zu jeweils 20 ausgewählt. Die Einteilung in die jeweilige Gruppe erfolgte nach dem Irregularity Index nach Little (1975). Dabei wurde entsprechend den Vorgaben zur Anwendung dieses Index vorgegangen. Dies bedeutet, dass die linearen Abstände der anatomischen Kontaktpunkte jedes unteren Frontzahnes zu den entsprechenden Punkten an seinen Nachbarzähnen mit einer Schieblehre (Zürcher Modell, Dental Liga, Köln, Deutschland) parallel zur Okklusionsebene gemessen werden. Dadurch erhält man ein Maß für die tatsächlichen Wegstrecken, die die anatomischen Kontaktpunkte aufeinander zu bewegt werden müssen, um eine ideale Zahnreihe zu bilden. Vertikale Abweichungen der Zähne blieben unberücksichtigt, da deren Korrektur die anteriore Zahnbogenlänge nicht signifikant beeinflusst. Bei Lücken und Rotationen wurde nur die vestibuloorale Verlagerung erfasst. Der Irregularitätsindex beträgt bei perfekter Anordnung der Zähne 0 .

Mit Hilfe des Irregularity Index wurden die Modelle in zwei Gruppen unterteilt (siehe Abbildung 1). Die erste Gruppe bestand aus Modellen des Unterkiefers mit einem Irregularity Index von 0-1, was keiner bis einer sehr geringen Irregularität der Frontzähne entspricht. Die zweite Gruppe setzte sich aus Modellen zusammen, deren Irregularity Index einem Wert von 4-10 entsprach. Dieser Wert weist auf eine moderate bis sehr starke Irregularität der Frontzähne hin. 
Jedes Modell wurde viermal kopiert und in Untergruppen A bis D eingeteilt. In Gruppe A wurde mit Hilfe eines herkömmlichen Übertragungskäppchens der Herstellungs- und Befestigungsprozess des 0,015 inch Dentaflex Retainers (Firma Dentaurum) untersucht.

Bei Gruppe B wurde die Magnetkette für die Herstellung und Befestigung eines 0,015 inch Dentaflex Retainers (Firma Dentaurum) verwendet. Mit Hilfe der Gruppen C und D wurde ein 0,018 inch Dentaflex (Firma Dentaurum) zur Herstellung und Befestigung des Retainers einmal mit konventioneller Übertragungstechnik (Gruppe C) und mit der Magnetkette (Gruppe D) untersucht.

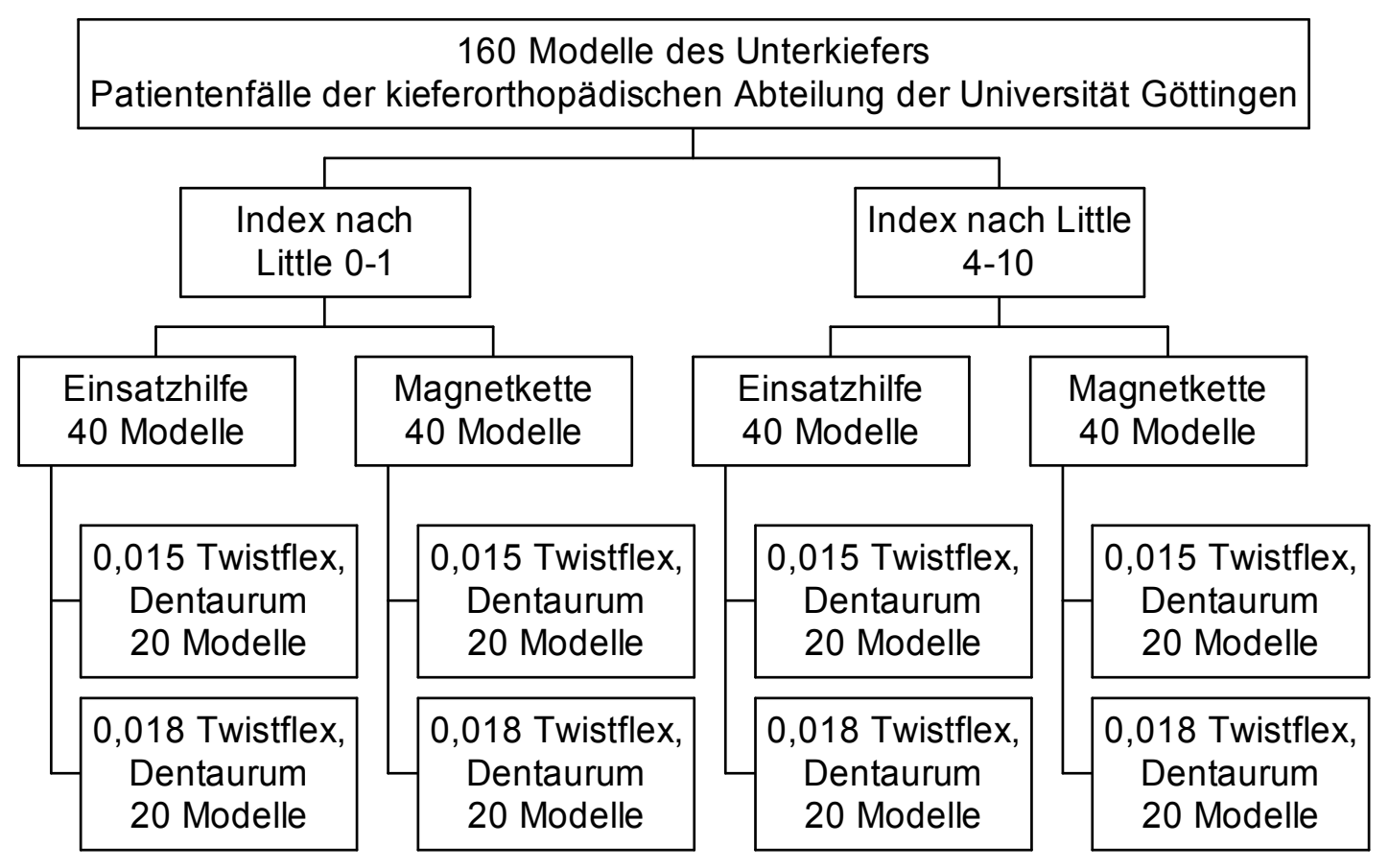

Abbildung 1: Aufteilung der Unterkiefermodelle in die zu untersuchenden Gruppen nach dem Irregularity Index nach Little

\subsubsection{Archivierung und Datenschutz}

Die Modelle wurden mit Hilfe von randomisiert gewählten Nummern pseudonymisiert und anhand des Gruppenbuchstabens den verschiedenen Gruppen zufällig zugeordnet. Patientendaten wurden nicht gespeichert. Die Originalmodelle der Abteilung für Kieferorthopädie wurden nur zur Herstellung von Dublierformen verwendet und anschließend für die Studie nicht weiter benötigt. 


\subsection{Modellherstellung}

Nach Auswahl der Modelle wurden nach Herstelleranweisung Dublierformen (additionsvernetztes Dubliersilikon, Adisil Blau, Siladent, Goslar, Deutschland) hergestellt. Diese dienten der Herstellung von vier identischen Kopien der ausgewählten Patientenmodelle aus Spezial-Hartgips Typ 3 (pico-crema soft, Picodent, Wipperfürth, Deutschland). Der Spezial-Hartgips wurde in einem Anmischverhältnis von 100 mg Pulver zu $30 \mathrm{ml}$ Wasser unter Vakuum (Multivac 4, Degussa Dental GmbH, Hanau, Deutschland) für 30 Sekunden angerührt.

Um Ungenauigkeiten der Sockelhöhe durch das Ausgießen auszugleichen, wurden aus einem Zwei-Komponenten-Knetsilikon (picodent twinduo, Picodent, Wipperfürth, Deutschland) Okklusionsschlüssel hergestellt. Mit Hilfe der Okklusionsschlüssel konnte jedes Patientenmodell reproduzierbar in einer individuell hergestellten Trimmhilfe positioniert und parallel zur Okklusionsebene getrimmt werden (Nasstrimmer Duo-Gipsmodelltrimmer 5350, Leleux Laborwelt, Mühlheim/Ruhr, Deutschland).

Anschließend wurden die Modelle mit Hilfe einer Einartikulierhilfe auf SAM-Montageplatten (SAM Präzisionstechnik GmbH, Gautling, Deutschland) befestigt. Durch die Einartikulierhilfe konnte sichergestellt werden, dass alle Modelle eines Patienten die gleiche räumliche Ausrichtung bekamen. Als Befestigungsmaterial wurde schnell abbindender Artikulationsgips (pico-arti speed, Picodent, Wipperfürth, Deutschland) in einem Anmischverhältnis von 100g Pulver auf $45 \mathrm{ml}$ Wasser verwendet. Der Gips wurde 15 Sekunden unter Vakuum angerührt.

\subsection{Retainer}

\subsubsection{Drähte}

In dieser Studie wurden zwei verschiedene Drahtqualitäten untersucht:

- 15 Dentaflex (Dentaurum, Ispringen, Deutschland)

- $\quad 18$ Dentaflex (Dentaurum, Ispringen, Deutschland)

Bei beiden Drahttypen handelt es sich um dreifach verseilte Drähte aus rostfreiem Stahl.

Der 15-Dentaflex weist einen Durchmesser von 0,38 mm (0,015 inch) auf. Der 18-Dentaflex hat einen Durchmesser von 0,45 mm (0,018 inch). Beide Drahtstärken haben einen Elastizitätsmodul von $170 \mathrm{kN} / \mathrm{mm}^{2}$. 


\subsubsection{Magnetkette}

Bei der Magnetkette handelt es sich um drei mit handelsüblichem Dentalkunststoff (WeiturPress, Johannes Weithas, Lütjenburg, Deutschland) gefasste $\mathrm{Ne}_{2} \mathrm{Fe}_{14} \mathrm{~B}$-Magnete (2 Zylinder $7 \times 3 \mathrm{~mm}, \mathrm{NdFeB}$ mit Nickelbeschichtung, 1.32 Tesla, 1 Zylinder $5 \times 2 \mathrm{~mm}, \mathrm{Nd}_{2} \mathrm{Fe}_{14} \mathrm{~B}$ mit Nickelbeschichtung, 1.29 Tesla, Neotexx, Berlin, Deutschland). Die $\mathrm{Nd}_{2} \mathrm{Fe}_{14} \mathrm{~B}-\mathrm{Magnete}$ werden durch nicht magnetisierbaren kieferorthopädischen Draht, zum Beispiel TMA-Drähte (Titan- Molybdän-Alloy-Draht), zu einer dreigliedrigen Kette verbunden (siehe Abbildung 2)

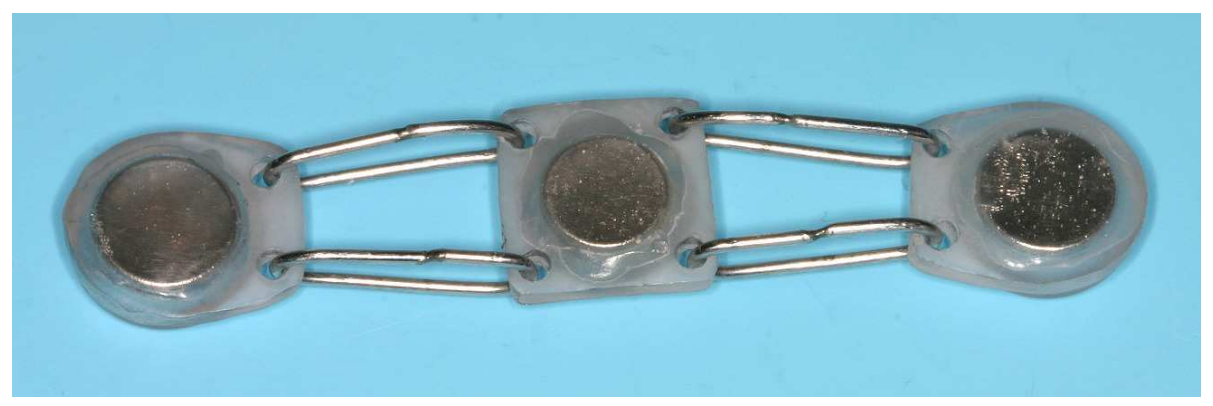

Abbildung 2: $\quad \mathrm{Ne}_{2} \mathrm{Fe}_{14} \mathrm{~B}$-Magnetkette mit Kunststoffummantelung

Die Länge der Kette soll der vestibulären Strecke der Zähne 33-43 entsprechen. Mit Hilfe von Abdämmwachs (Surgident Periphery-Wachs, Heraeus Kulzer, Hanau, Deutschland) kann die Magnetkette an den vestibulären Flächen der Zähne befestigt werden (siehe Abbildung 3).

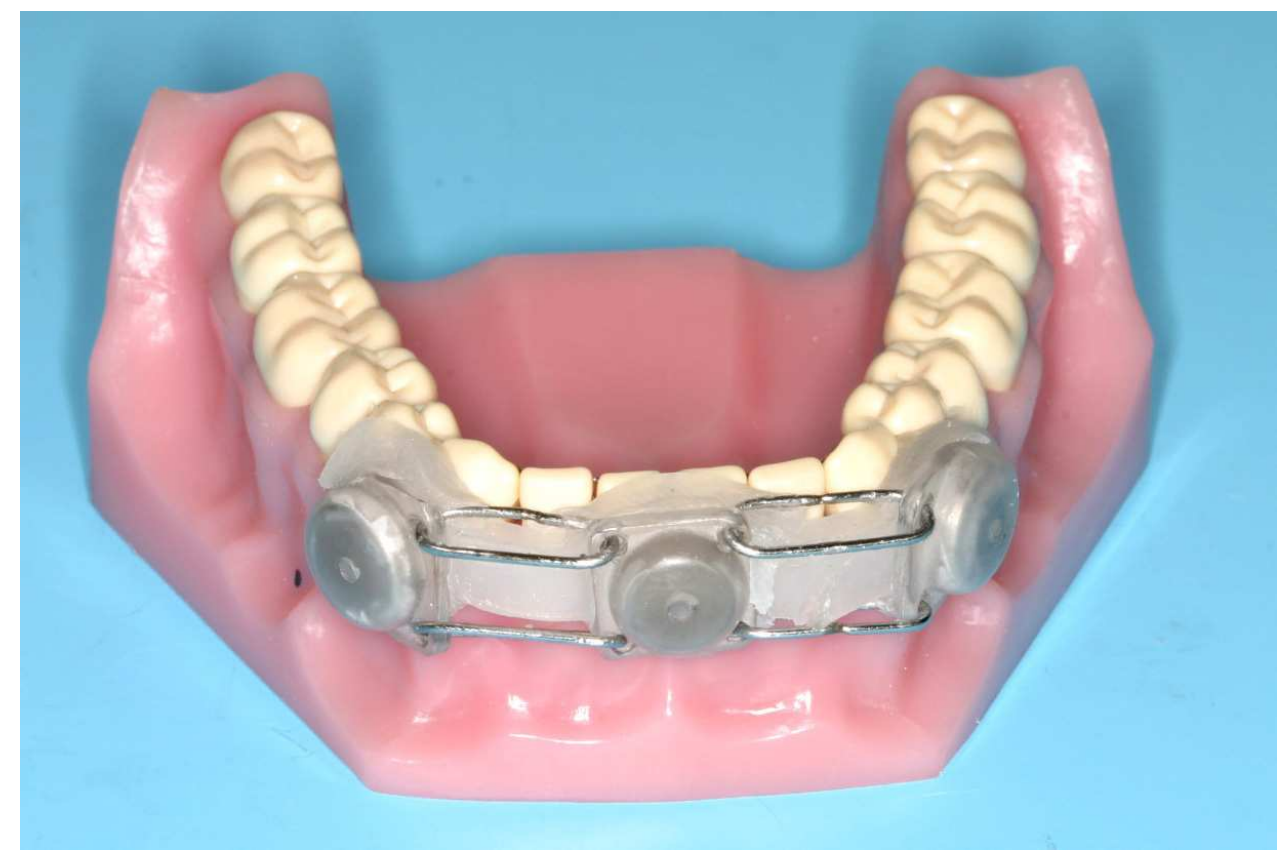

Abbildung 3: Die $\mathrm{Ne}_{2} \mathrm{Fe}_{14} \mathrm{~B}$-Magnetkette wird mit Abdämmwachs an den vestibulären Flächen der Zähne befestigt 
Durch das sich auch auf der lingualen Seite der Zähne entwickelte Magnetfeld wird der Draht des 3-3-Retainers an die Zahnoberfläche gezogen und dort gehalten.

\subsubsection{Herstellung der Retainer}

Die Retainer wurden von einem Zahntechniker der Abteilung Kieferorthopädie der Universitätsmedizin Göttingen auf den pseudonomisierten Modellen innerhalb einer Gruppe randomisiert hergestellt. Die Herstellung der Retainer erfolgte durch die konventionelle Technik mit Einsatzhilfe und mit der Magnettechnik. Die Arbeitsschritte wurden anhand einer Arbeitsanweisung (siehe Anhang) für den Zahntechniker festgelegt. Die benötigte Zeit jedes Herstellungsprozesses wurde gemessen (Stoppuhr HS-3V-1RET, Casio, Norderstedt, Deutschland) und in der Arbeitsanweisung für jedes Modell dokumentiert.

\subsubsection{Herstellung eines Retainers mit Einsatzhilfe}

Die Zeitmessung zur Herstellung eines 3-3-Retainers durch die konventionelle Technik mit Einsatzhilfe beginnt mit dem Isolieren der Gipsmodelle mit Isolant (Dentsply, Konstanz, Deutschland), einem Isolationsmittel für Gips gegen Kunststoff. So soll verhindert werden, dass sich die aus Kunststoff hergestellte Einsatzhilfe mit dem Gipsmodell verbindet und ein Ablösen von dem Modell nicht möglich ist. Die Überschüsse des Isolationsmittels wurden entfernt, und aus dem jeweiligen Draht (0,015 inch Dentaflex bzw. 0,018 inch Dentaflex, Dentaurum, Ispringen, Deutschland) wurde entlang der lingualen Oberfläche der Schneideund Eckzähne ein Drahtbogenretainer angepasst. Nach Anpassung des Drahtbogenretainers wurde dieser mit Wachs (Surgident Periphery-Wachs, Heraeus Kulzer, Hanau, Deutschland) an den Eckzähnen des Modells befestigt, und es folgte die Herstellung der Einsatzhilfe. Hierfür wurde aus Vita VMLC lichthärtendem Kunststoff (Vita, Bad Säckingen, Deutschland) ein circa $5 \mathrm{~mm}$ x $10 \mathrm{~mm}$ großes Rechteck geformt, welches zwischen den ersten Inzisivi von labial nach lingual über die Inzisalkante an den Zahnoberflächen der Gipszähne sowie den Drahtbogen in dieser Region adaptiert wurde (siehe Abbildung 4). 


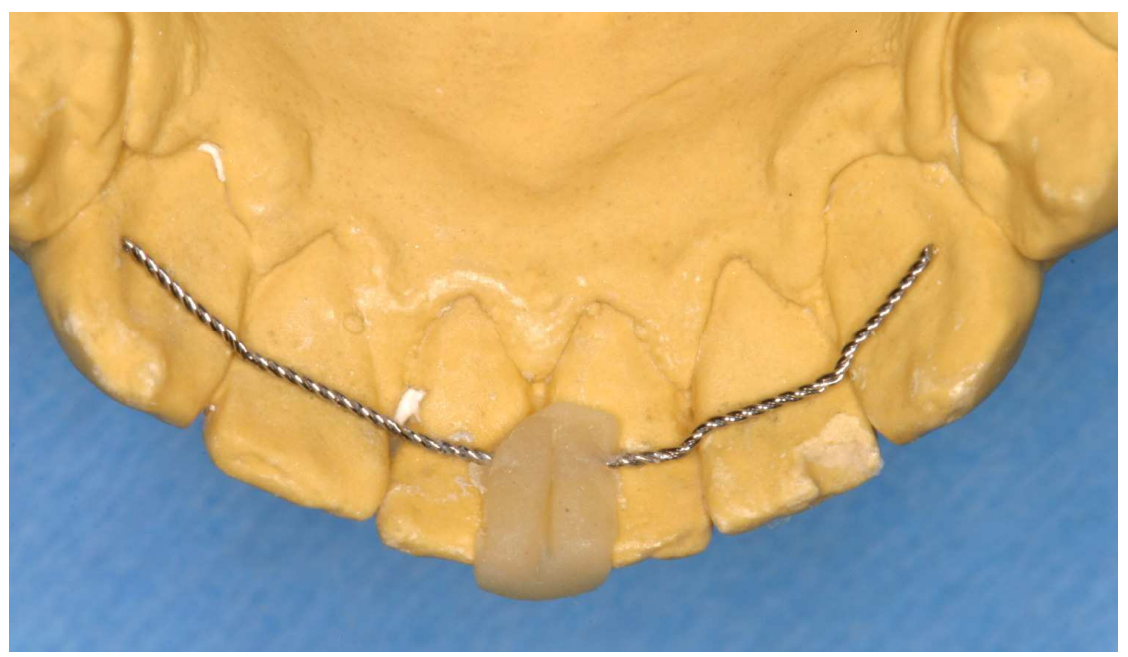

Abbildung 4: $\quad$ Modell mit 3-3-Retainer durch Einsatzhilfe fixiert

Nachdem überschüssiger Kunststoff entfernt wurde, folgte die Aushärtung des Kunststoffes mittels einer Polymerisationslampe (Astralis 5, Vivadent, Lichtenstein, Österreich) für 30 Sekunden. Anschließend wurde das Wachs mit Hilfe eines Dampfstrahlgerätes (Aquaclean 3, Degussa Dental GmbH, Hanau, Deutschland) entfernt und der Retainer mit der Einsatzhilfe vom Modell genommen. Danach erfolgte die Kontrolle des Retainers mit Einsatzhilfe auf seine Passung sowie einen spannungsfreien Sitz und eventuelle Korrekturen. Die Zeitmessung endete zu dem Zeitpunkt, an dem der Techniker den Retainer mit Einsatzhilfe für einsatzfähig erklärte.

\subsubsection{Herstellung eines Retainers mit Magnetkette}

Die Herstellung eines 3-3-Retainers mit der Magnetkette startete mit der Befestigung der Magnetkette an den labialen Oberflächen der Frontzähne mit Hilfe von Wachs (Surgident Periphery Wax, Heraeus Kulzer, Hanau, Deutschland). Anschließend wurde der Retainer aus dem entsprechenden Draht (0,015 inch Dentaflex bzw. 0,018 inch Dentaflex, Dentaurum Ispringen, Deutschland) angepasst (siehe Abbildung 5). 


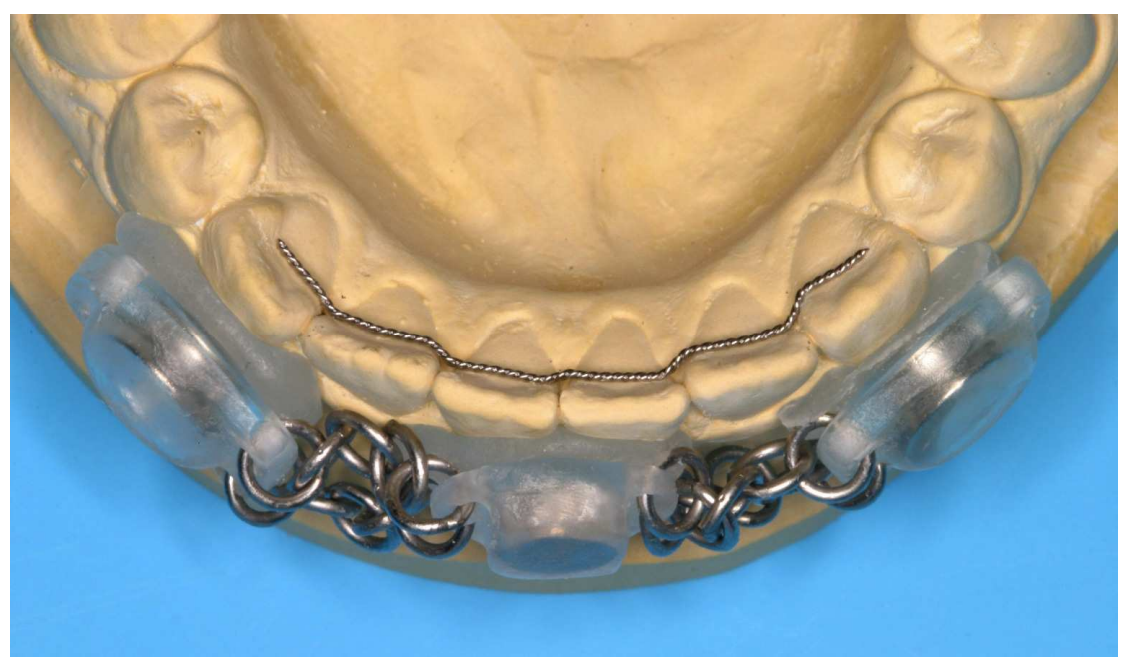

Abbildung 5: $\quad$ Modell mit 3-3-Retainer durch Magnetkette fixiert (Abbildung aus Hahn et al. 2011, S. 383)

Nach Herstellung des Drahtbogenretainers wurde seine Passung sowie ein spannungsfreies Anliegen an die lingualen Zahnoberflächen durch den Zahntechniker kontrolliert und eventuelle Korrekturen vorgenommen. Mit der Entfernung der Magnetkette und des Wachses vom Gipsmodell endete die Zeitmessung für den Produktionsprozess.

\subsection{Adhäsive Befestigung}

Nach Herstellung aller Retainer wurden die pseudonomisierten Modelle in dentalen Simulationseinheiten (KaVo Dental GmbH, Biberach/Riß, Deutschland) befestigt, um den adhäsiven Befestigungsvorgang möglichst praxisnah durchzuführen. Anschließend wurden die Retainer von einem Behandler der Abteilung für Kieferorthopädie der Universitätsmedizin Göttingen mittels Adhäsivtechnik randomisiert geklebt. Die adhäsive Befestigung mit Hilfe der Einsatzhilfe oder der Magnetkette erfolgte nach den Vorgaben einer entsprechenden Arbeitsanweisung (siehe Anhang) für alle Modelle gleich. Für jeden Klebevorgang wurde die benötigte Zeit in Minuten [min] gestoppt und dokumentiert. Um eine Lernkurve des Behandlers bei den Messungen auszuschließen, wurden vor der Messung alle Arbeitsschritte an zehn Modellen geübt.

\subsubsection{Adhäsive Befestigung eines Retainers mit Einsatzhilfe}

Bei der adhäsiven Befestigung eines 3-3-Retainers mit Einsatzhilfe begann die Zeitmessung mit dem Aufnehmen des Retainers vom Tray durch den Behandler. Der Retainer wurde auf das Modell gesetzt, ausgerichtet und auf seine Passgenauigkeit überprüft. Anschließend wurde 
der Retainer wieder vom Modell entfernt. Die adhäsive Befestigung des Retainers begann mit dem Auftragen des Adhäsivs Transbond XT primer (3M Unitek, Monrovia, California, USA), welches anschließend verpustet und für $30 \mathrm{sec}$. mit einer Polymerisationslampe (Astralis 5, Ivoclar Vivadent, Ellwangen Jagst, Deutschland) ausgehärtet wurde. Nach Auftragen des Adhäsivs wurde der Retainer wieder auf das Modell gesetzt und mit Hilfe des fließfähigen Kunststoffes Transbond LR (3M Unitek, Monrovia, California, USA) an den Zähnen 33 bis 43 lingual befestigt. Der Befestigungskunststoff wurde ebenfalls für 30 Sekunden in jedem Quadranten gehärtet. Da die Einsatzhilfe auch die linguale Fläche der ersten Inzisivi bedeckt, konnten gelegentlich die ersten Inzisivi erst nach Entfernung der Einsatzhilfe in die Klebung mit einbezogen werden. Nach Entfernung der Einsatzhilfe endete die Zeitmessung.

\subsubsection{Adhäsive Befestigung eines Retainers mit Magnetkette}

Die Zeitmessung des Klebevorgangs mit Hilfe der Magnetkette wurde in drei Schritte unterteilt.

Zunächst wurde das Aufbringen der in einer Wachsstange (Surgident Periphery-Wachs, Heraeus Kulzer, Hanau, Deutschland) befestigten Magnetkette an die labialen Flächen der Gipszähne gemessen. Anschließend wurde der Klebevorgang gemessen. Die Zeiterfassung begann mit dem Aufnehmen des Retainers vom Tray. Anschließend wurde der Retainer auf das Modell aufgesetzt und bis zur optimalen Passung in vertikaler und horizontaler Richtung ausgerichtet. Daraufhin wurde der Retainer wieder abgenommen und das Adhäsiv Transbond XT primer (3M Unitek) aufgetragen, welches 30 Sekunden mit einer Polymerisationslampe in jedem Quadranten ausgehärtet wurde. Anschließend wurde der Retainer erneut aufgesetzt und der Kunststoff Transbond LR (3M Unitek) auf jedem Zahn in einem Arbeitsgang lingual aufgetragen. Die Zeitmessung endet nach dem Aushärten des Kunststoffs für jeweils 30 Sekunden in jedem Quadranten.

Im letzten Schritt wurde die Zeit, die zum vollständigen Entfernen der Magnetkette sowie des Wachses von den labialen Flächen der Gipszähne benötigt wurde, gemessen.

\subsection{Weiterbearbeitung der Modelle}

Die mit den 3-3-Retainern versehenen pseudonymisierten Modelle wurden von den SAMMontageplatten entfernt. Anschließend wurde durch Trimmen der Modelle an einem Feintrimmer das Segment zwischen den jeweiligen Eckzähnen herausgelöst. Danach erfolgte die Reduzierung der mit dem Retainer versehenen Gipszähne in der Vertikalen bis auf Höhe des Retainerdrahtes (siehe Abbildung 6). 


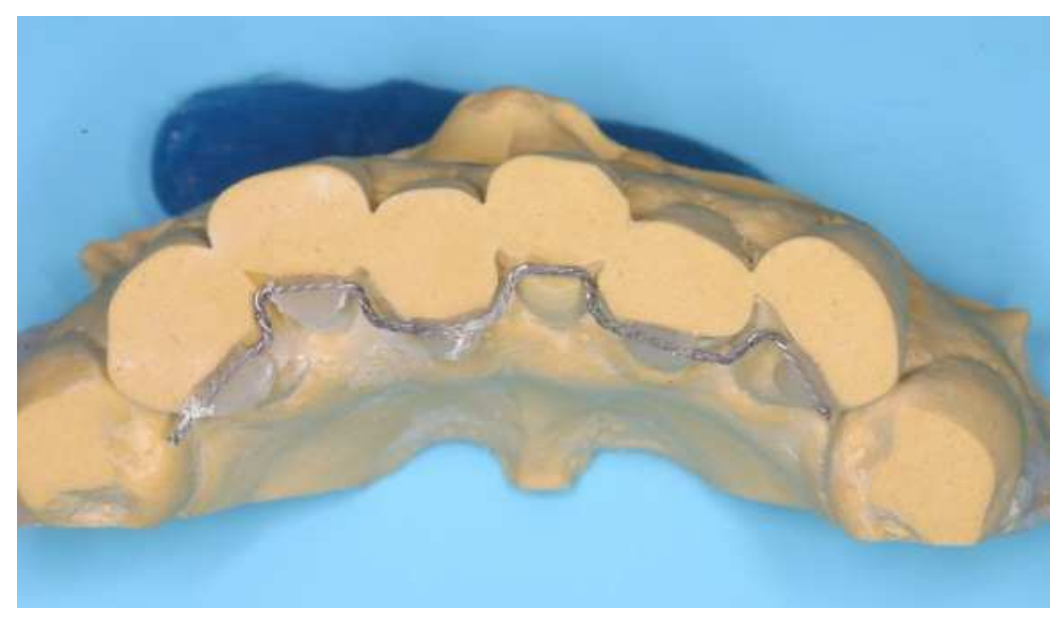

Abbildung 6: Das Front-Eckzahnsegment wurde aus dem Modell herausgelöst und in der Vertikalen parallel bis auf Höhe des Retainerdrahtes reduziert.

Die so entstandenen Segmente wurden auf Ebene des Retainers mit Hilfe eines Flachbildscanners (Epson Perfection V750 pro, Epson Deutschland GmbH, Meerbusch, Deutschland) mit einer Auflösung von 3200 dpi eingescannt.

\subsubsection{Auswertung der Bilddateien}

Die durch das Einscannen der Modelle erhaltenen Bilddateien wurden mit Hilfe des automatischen Bildanalysesystems KS 300 (Carl Zeiss, Jena, Deutschland) vermessen (siehe Arbeitsanweisung im Anhang). Pro Bild wurden zehn Messwerte erhoben. Zunächst wurde die Fläche zwischen Zahnbogen und Drahtbogen $\left[\mathrm{mm}^{2}\right]$ gemessen. Um Ungenauigkeiten durch verschiedene Drahtlängen, die durch den Herstellungsprozess entstehen können, entgegenzuwirken, wurde bei der Messung der Fläche das letzte Drittel des Eckzahnes in seiner mesio-distalen Ausdehnung nicht berücksichtigt (siehe Abbildung 7). 


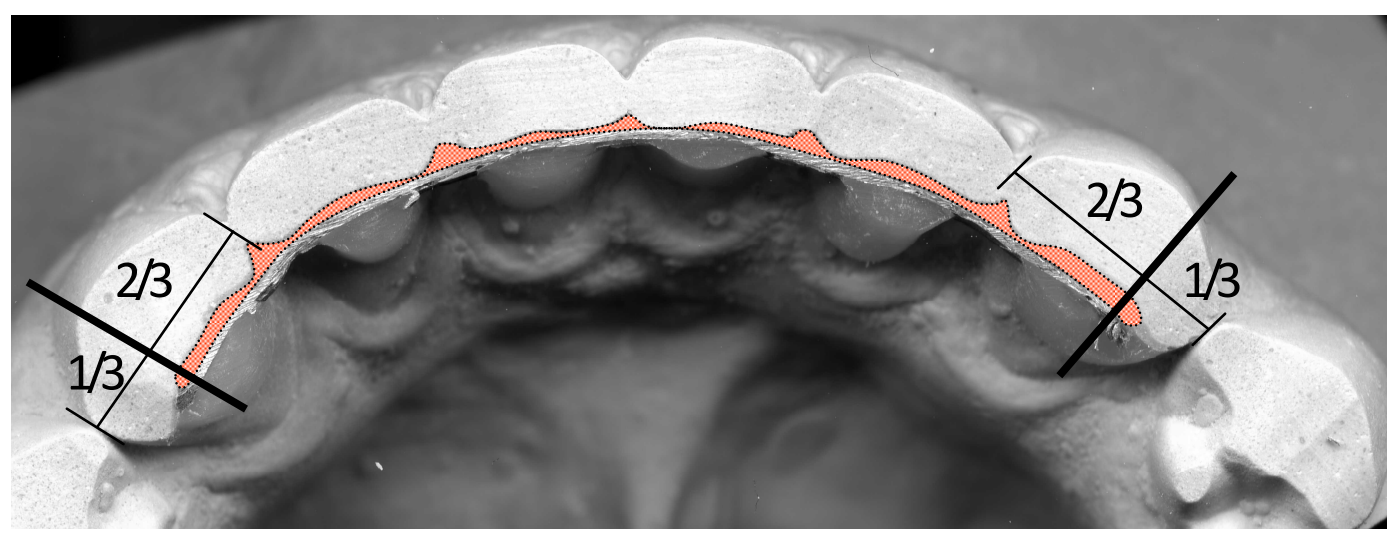

Abbildung 7: Ausgemessene Fläche $\left[\mathrm{mm}^{2}\right]$ (rot markierte Fläche) zwischen lingualer Zahnoberfläche und labialer Begrenzung des Retainerdrahtes. Das letzte Drittel des endständigen Eckzahnes in seiner mesio-distalen Ausdehnung wurde bei der Messung nicht berücksichtigt.( Abbildung aus Hahn et al. 2011, 384)

Außerdem wurde der senkrechte Abstand [mm] zwischen der oralen Kante eines jeden beklebten Zahnes in der Mitte seiner mesio-distalen Ausdehnung senkrecht zu einer Tangente der nach labial gerichteten Kante des Retainerdrahtes eines jeden beklebten Zahnes gemessen (siehe Abbildung 8).

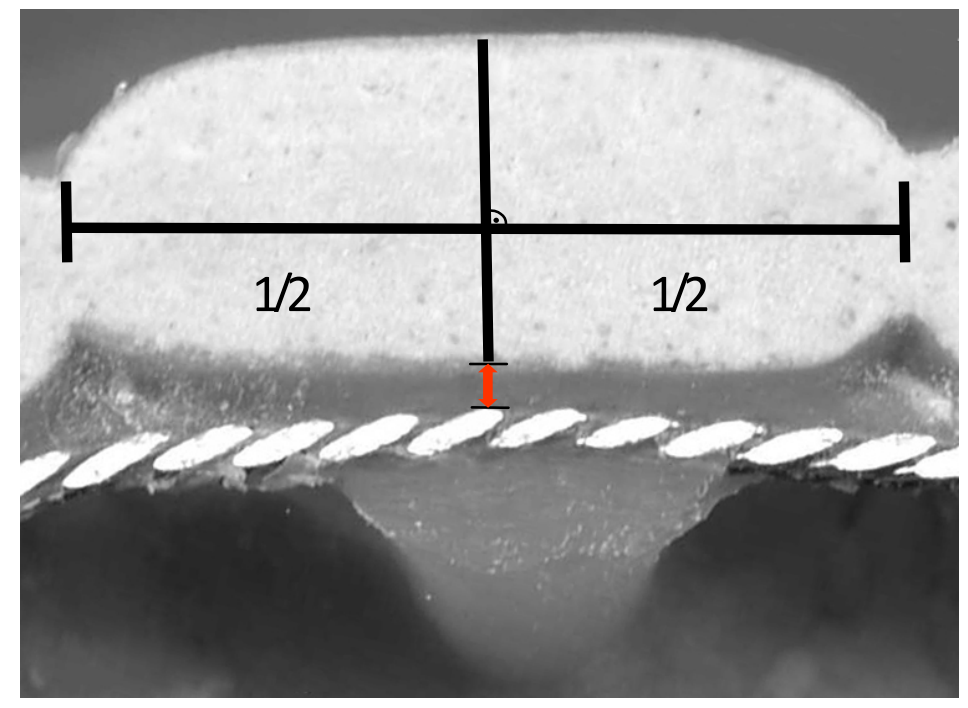

Abbildung 8: Gemessen wurde der senkrechte Abstand [mm] (rot markiert) zwischen lingualer Zahnoberfläche und labialer Begrenzung des Retainerdrahtes (Abbildung aus Hahn et al. 2011, S. 384) 
Alle Messungen wurden durch einen Untersucher durchgeführt. Jedes Bild wurde zweimal vermessen.

Alle Messwerte wurden mit Hilfe des Programms Excel 2007 (Microsoft, Redmon, WA, USA) tabellarisch erfasst.

\subsection{Statistik}

Die statistische Auswertung erfolgte in Zusammenarbeit mit der Abteilung Medizinische Statistik der Universität Göttingen.

Die Güte der Anpassung des Drahtes an die Zähne sollte primär über die Fläche $\left[\mathrm{mm}^{2}\right]$ zwischen Zahn- und Drahtbogen verglichen werden. Als Sekundärhypothese wurde diese Größe aufgespalten in die Distanzen [mm] der inzisal-oralen Kante eines jeden Zahnes zur labialen Kante des Retainerdrahtes.

Des Weiteren wurden Biegezeit und Befestigungszeit beider Methoden verglichen.

Die Analyse der Daten erfolgte mittels einer ANOVA. 


\section{$4 \quad$ Ergebnisse}

\subsection{Auswertung der Fläche}

Mit Hilfe des Bildanalysesystems KS 300 (Carl Zeiss, Jena, Deutschland) erfolgte die Messung der Fläche zwischen Drahtbogen und Zahnbogen in $\left[\mathrm{mm}^{2}\right]$. Gemessen wurde die Fläche zwischen der oralen Kante der Zähne 33-43 und der nach labial orientierten Kante des Retainerdrahtes.

In Abbildung 9 ist die Auswertung der Fläche grafisch dargestellt.

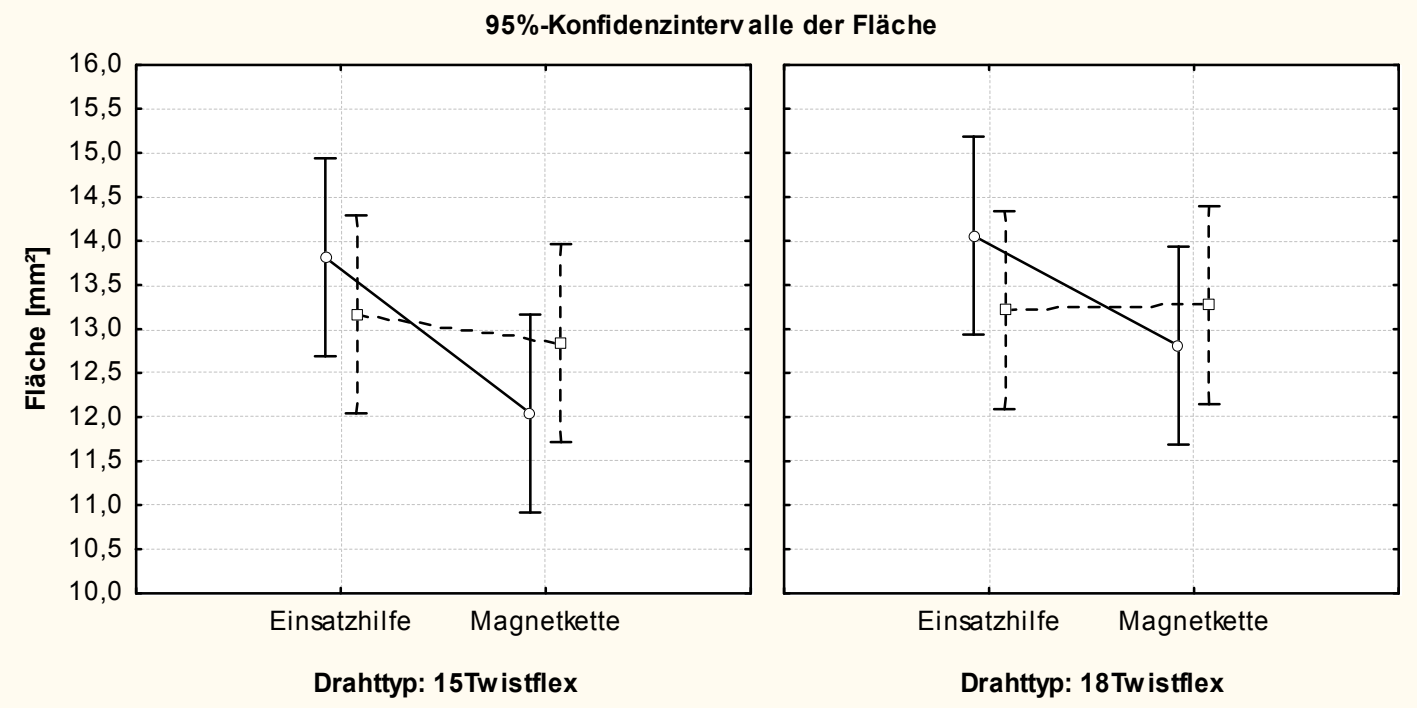

Abbildung 9: Gemessen wurde die Fläche zwischen der lingualen Kante der Zähne 33-43 und des Retainerbogens bei Verwendung eines 15er-Twistflex-Drahtes (links) und eines 18er-Twistflex-Drahtes (rechts). Verglichen wurden die Standardmethode mit Einsatzhilfe und die neue Methode mit Magnetkette. Die Methoden wurden jeweils bei 20 Unterkiefermodellen mit einem Index nach Little von 0-1 (keine bis geringe Irregularität) und einem Index nach Little von 4-10 (moderate bis starke Irregularität) durchgeführt. Die Flächenwerte werden in $\left[\mathrm{mm}^{2}\right]$ angegeben.

durchgängige Linie = Index nach Little 0-1 gestrichelte Linie = Index nach Little 4-10

In der linken Grafik in Abbildung 9 werden der Mittelwert sowie das 95\%-Konfidenzintervall der Fläche $\left[\mathrm{mm}^{2}\right]$ bei Verwendung eines Retainers aus 15er Twistflex dargestellt. Bei der Modellgruppe Index nach Little 0-1 liegt der Mittelwert für die Standardmethode bei 13,81 $\mathrm{mm}^{2}$ [95\%-Konfidenzintervall 12,68 $\left.\mathrm{mm}^{2}-14,94 \mathrm{~mm}^{2}\right]$. Der Mittelwert für die Methode mit 
der Magnetkette liegt bei 12,04 $\mathrm{mm}^{2}$ [95\%-Konfidenzintervall 10,92 mm² - 13,16 $\mathrm{mm}^{2}$ ]. Bei der Modellgruppe Index nach Little 4-10 lag der Mittelwert für die Standardmethode bei $13,17 \mathrm{~mm}^{2}$ [95\%-Konfidenzintervall 12,04 $\left.\mathrm{mm}^{2}-14,29 \mathrm{~mm}^{2}\right]$.

In der rechten Grafik in Abbildung 9 werden der Mittelwert sowie das 95\%Konfidenzintervall für die Fläche $\left[\mathrm{mm}^{2}\right]$ zwischen Drahtbogen und Zahnbogen bei Verwendung eines Retainers aus 18er Twistflex dargestellt. Bei den Modellen mit einem Index nach Little von 0-1 kann bei der Standardmethode ein Mittelwert von 14,06 mm² [95\%Konfidenzintervall 12,94 $\mathrm{mm}^{2}-15,17 \mathrm{~mm}^{2}$ ] errechnet werden. Für die Methode mit der Magnetkette ergibt sich ein Wert von 12,81 $\mathrm{mm}^{2}$ [95\%-Konfidenzintervall 11,68 mm² - 13,93 $\mathrm{mm}^{2}$ ]. In der Modellgruppe Index nach Little 4-10 zeigt sich ein Mittelwert von 13,21 $\mathrm{mm}^{2}$ [95\%-Konfidenzintervall 12,09 $\mathrm{mm}^{2}-14,33 \mathrm{~mm}^{2}$ ] für die Standardmethode und ein Mittelwert von 13,27 $\mathrm{mm}^{2}$ [95\%-Konfidenzintervall $\left.12,15 \mathrm{~mm}^{2}-4,38 \mathrm{~mm}^{2}\right]$ für die neue Technik mit Magnetkette.

Der statistische Vergleich zeigt, dass die Fläche zwischen Zahn- und Drahtbogen bei Verwendung der Magnetbandmethode signifikant kleiner ist als bei der Methode mit Einsatzhilfe (p-Wert=0,0347). Der Unterschied zwischen beiden Methoden (Magnetkettenmethode vs. Methode mit Einsatzhilfe) wird auf $0,8234 \mathrm{~mm}^{2}$ [95\%Konfidenzintervall $0,0287 \mathrm{~mm}^{2}-0,6180 \mathrm{~mm}^{2}$ ] geschätzt.

Ein Einfluss der Gruppe nach dem Irregularitätsindex nach Little (p-Wert 0,8321) sowie des Drahttyps (p-Wert 0,2557) auf die Fläche ist nicht zu erkennen.

\subsection{Auswertung des senkrechten Abstandes}

Der senkrechte Abstand [mm] wurde definiert als die Strecke zwischen der oralen Kante eines jeden beklebten Zahnes in der Mitte seiner mesio-distalen Ausdehnung senkrecht zu einer Tangente der nach labial gerichteten Kante des Retainerdrahtes eines jeden beklebten Zahnes. In Abbildung 10 werden der Mittelwert sowie das 95\%-Konfidenzintervall des senkrechten Abstandes [mm] der oralen Kante der Zähne 33, 32, 31, 41, 42, 43 zur labialen Kante des Retainers aus 15 er Twistflex grafisch dargestellt. Abbildung 11 stellt den Mittelwert sowie das 95\%-Konfidenzintervall des senkrechten Abstandes [mm] der oralen Kante der Zähne 33, 32, 31, 41, 42, 43 zur labialen Kante des Retainers aus 18 er Twistflex grafisch dar. 
95\%-Konfidenzintervall Distanz Drahttyp: Tw istflex15

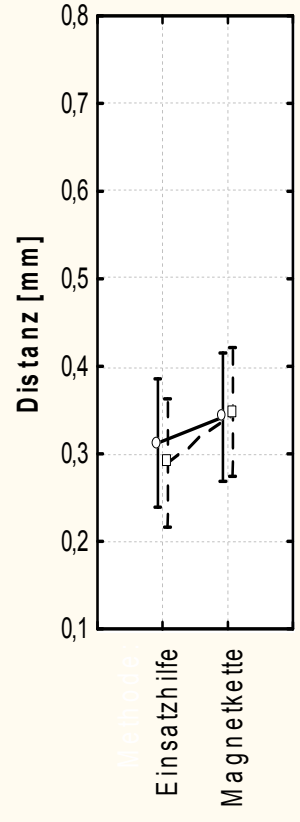

Zahn 33

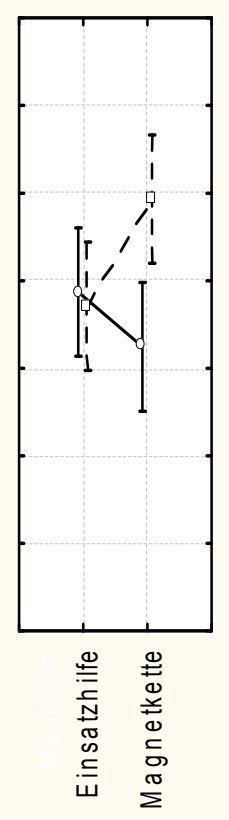

Zahn 32
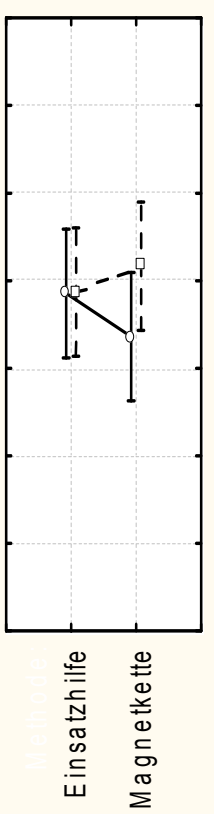

Zahn 31
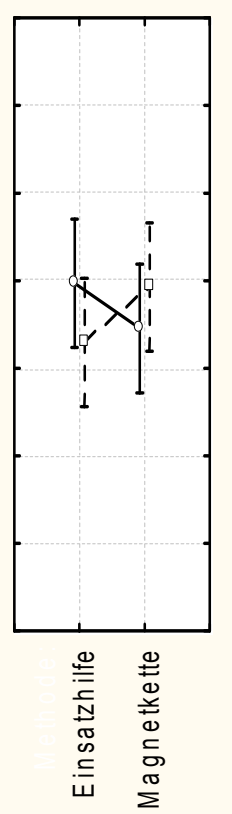

Zahn 41

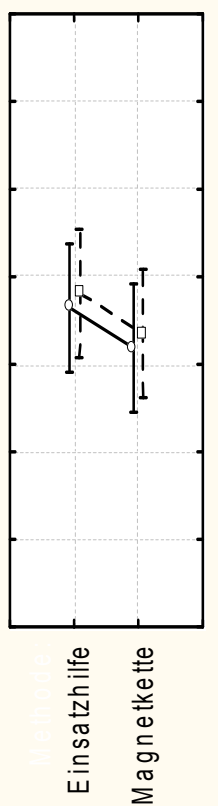

Zahn 42

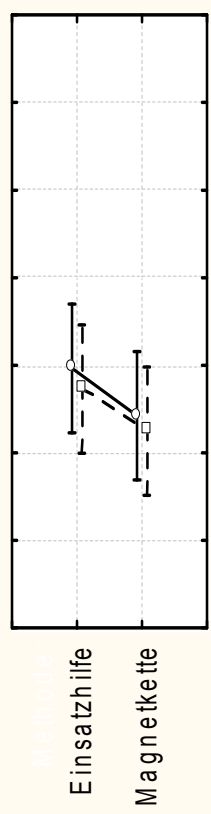

Zahn 43

Abbildung 10: Gemessen wurde der senkrechte Abstand [mm] der oralen Kante der Zähne 33, 32, 31, 41, 42, 43 zur labialen Kante eines 15er Twistflex-Drahtes (siehe Abb. 8, Material und Methoden). Verglichen wurden die Methode mit Einsatzhilfe und die Methode mit Magnetkette. Die Methoden wurden jeweils an 20 Unterkiefermodellen mit einem Irregularity-Index nach Little von 0-1 (keine bis geringe Irregularität) und einem Irregulaity-Index nach Little von 4-10 (moderate bis starke Irregularität) untersucht.

durchgängige Linie = Index nach Little 0-1

gestrichelte Linie = Index nach Little 4-10

Die Gesamtheit der Messwerte des senkrechten Abstandes [mm] lagen im Mittel für die Modellgruppe mit einem Irregularity-Index nach Little 0-1 zwischen 0,31 mm (Zahn 33, Neodym-Magnetkette, 18er-Twistflex) und 0,517 $\mathrm{mm}$ (Zahn 32, Einsatzhilfe, 18erTwistflex). Für die Modellgruppe mit einem Irregularity-Index nach Little 4-10 lagen die Mittelwerte zwischen 0,29 mm (Zahn 33, Einsatzhilfe, 15er-Twistflex) und 0,593 mm (Zahn 32, Magnetkette, 15er Twistflex).

Wurde ein Retainer aus 15er-Twistflex mit einer Einsatzhilfe bei einem Modell der Gruppe Irregularity-Index nach Little 0-1 geklebt, konnte der kleinste senkrechte Abstand an Zahn 33 mit einem Mittelwert von 0,313 mm (95\%-Konfidenzintervall 0,241 mm - 0,384 mm) gemessen werden. Der Maximalwert lag mit einem Mittelwert von 0,497 mm (95\%- 
Konfidenzintervall 0,424 mm - 0,567 mm) an Zahn 41. Wurde bei der gleichen Modellgruppe ein Retainer aus 15er-Twistflex mit Hilfe der Magnetkette geklebt, lag der Minimalwert für den senkrechten Abstand an Zahn 33 mit einem Mittelwert von 0,342 (95\%Konfidenzintervall 0,270 mm - 0,414 mm). Der Maximalwert für die Methode mit der Magnetkette konnte an Zahn 41 mit einem Mittelwert von 0,446 mm (95\%Konfidenzintervall 0,374 mm - 0,517 mm) gemessen werden.

Nach adhäsiver Befestigung von Retainern aus 18er-Twistflex bei Modellen der Gruppe Irregularity-Index nach Little 0-1 konnte bei Verwendung der Einsatzhilfe der geringste senkrechte Abstand an Zahn 33 mit einem Mittelwert von 0,363 mm (95\%-Konfidenzintervall mm 0,291 - 0,434 mm) gemessen werden. Der größte senkrechte Abstand lag mit einem Mittelwert von 0,517 (95\%-Konfidenzintervall 0,445 mm - 0,589 mm) an Zahn 32. Wurde für die Fixierung eines 18er-Twistflex Retainers an Modellen der Gruppe Irregularity-Index nach Little 0-1 die Neodym-Magnetkette verwendet, so wurde mit einem Mittelwert von 0,310 mm (95\%-Konfidenzintervall 0,238 mm - 0,381 mm) der kleinste senkrechte Abstand an Zahn 33 und mit einem Mittelwert von 0,439 mm (95\%-Konfidenzintervall 0,367 mm - 0,511 mm) der größte senkrechte Abstand an Zahn 31 gemessen.

Nach adhäsiver Befestigung von Retainern aus 15er-Twistflex mit Hilfe der Einsatzhilfe an Modellen der Gruppe Irregularity-Index nach Little 4-10 wurde der kleinste senkrechte Abstand mit einem Mittelwert von 0,290 mm (95\%-Konfidenzintervall 0,218 mm - 0,361 $\mathrm{mm}$ ) an Zahn 33 und der größte senkrechte Abstand mit einem Mittelwert von 0,487 mm (95\%-Konifendzintervall 0,415 mm - 0,559 mm) an Zahn 31 gemessen. Bei Verwendung desselben Drahttyps und derselben Modellgruppe konnte bei Fixierung der Retainer mit der Neodym-Magnetkette ein Minimalwert des senkrechten Abstandes mit einem Mittelwert von 0,325 mm (95\%-Konfidenzintervall 0,253 mm - 0,397 mm) an Zahn 42 gemessen werden.

Der Maximalwert wurde an Zahn 32 mit einem Mittelwert von 0,593 (95\%Konfidenzintervall 0,521 mm - 0,665 mm) gemessen.

Wurde der Retainer bei der Modellgruppe Irregularity-Index nach Little 4-10 aus 18er Twistflex gefertigt und mit der Einsatzhilfe fixiert, so konnte der senkrechte Abstand an Zahn 33 mit einem Mittelwert von 0,303 mm (95\%-Konfidenzintervall 0,231 mm - 0,375 mm) als Minimalwert gemessen werden. Der Maximalwert lag für diesen Drahttyp und der Methode mit Einsatzhilfe an Zahn 32 mit einem Mittelwert von 0,482 mm (95\%-Konfidenzintervall 0,410 mm - 0,553 mm). Wurde zur Positionierung und Fixierung des Retainers aus 18er Twistflex bei der Modellgruppe Irregularity-Index nach Little 4-10 die Neodym-Magnetkette verwendet, so lag der geringste senkrechte Anstand an Zahn 33 mit einem Mittelwert von 
0,426 mm (95\%-Konfidenzintervall 0,354 mm - 0,496 mm). Der größte senkrechte Abstand wurde mit einem Mittelwert von 0,497 mm (95\%-Konfidenzintervall 0,424 mm - 0,569 mm) an Zahn 31 gemessen.

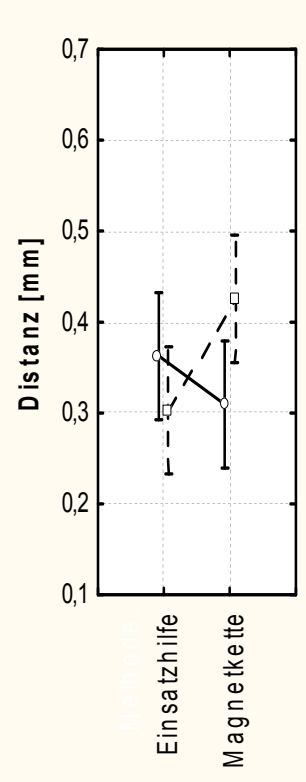

Zahn 33

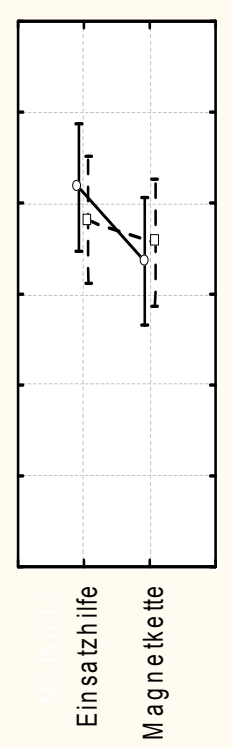

Zahn 32
95\%-Konfidenzintervall Tw istflex 18

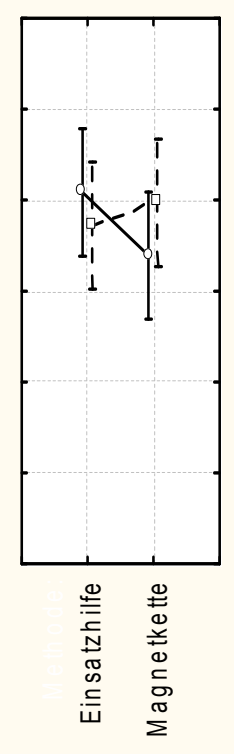

Zahn 31

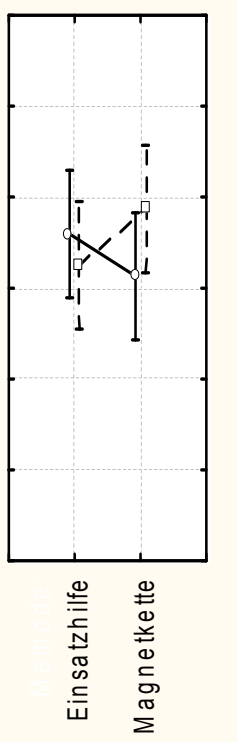

Zahn 41

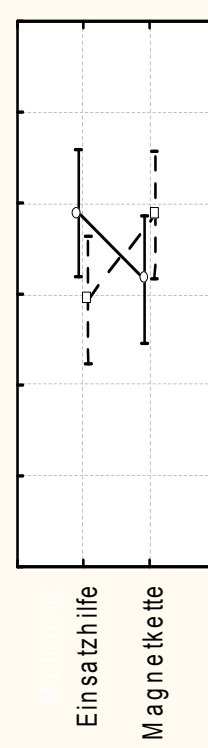

Zahn 42

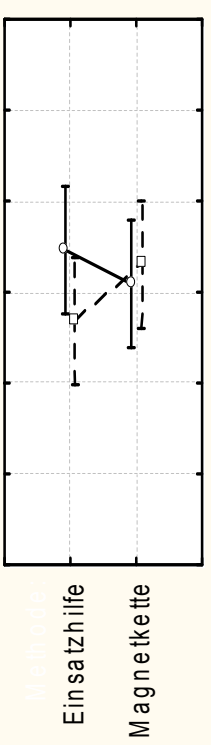

Zahn 43

Abbildung 11: Gemessen wurde der senkrechte Abstand [mm] der inzisal-oralen Kante der Zähne 33, 32, 31, 41, 42, 43 zur labialen Kante eines 18er Twistflex-Drahtes. Verglichen wurden die Standardmethode mit Einsatzhilfe und die neue Methode mit Magnetkette. Die Methoden wurden jeweils an 20 Unterkiefermodellen mit einem Index nach Little von 0-1 (keine bis geringe Irregularität) und einem Index nach Little von 4-10 (moderate bis starke Irregularität) untersucht.

durchgängige Linie = Index nach Little 0-1 gestrichelte Linie = Index nach Little 4-10

Es zeigte sich, dass der Drahttyp keinen signifikanten Einfluss auf den senkrechten Abstand hat (p-Wert 0,2615). Die Lokalisation der Messung im Unterkiefer (Zahn33 bis Zahn 43) beeinflusst signifikant den gemessenen senkrechten Abstand ( $p$-Wert $<0,0001$ ). Des Weiteren ist die Wechselwirkung Gruppe*Methode ( $\mathrm{p}$-Wert $<0,0001)$ signifikant. Dies bedeutet, dass der Unterschied zwischen beiden angewendeten Methoden abhängig davon ist, an welcher 
Modellgruppe die jeweilige Methode angewendet wurde. Als Folge wurde der Datensatz nach Gruppe Irregularity-Index nach Little 0-1 und Gruppe Irrgularity-Index nach Little 4-10 aufgespalten. Für jede Gruppe wurde eine separate Analyse unabhängig von der Drahtstärke durchgeführt.

Betrachtet man den senkrechten Abstand [mm] der lingualen Kante der Zähne 33, 32, 31, 41, 42, 43 zur labialen Kante eines 3-3- Retainers unabhängig von der Drahtstärke, so ergab sich in der Modellgruppe Index nach Little 0-1 ein signifikanter Einfluss der Methode auf die gemessene Distanz ( $\mathrm{p}$-Wert $<0,0002$ ). Der Unterschied zwischen der Standardmethode mit Einsatzhilfe und der neuen Methode mit Magnetkette wird im Mittel auf 0,04950 mm (95\%Konfidenzintervall $0,02260 \mathrm{~mm}-0,07640 \mathrm{~mm}$ ) geschätzt. Dies bedeutet, dass bei der Methode mit Magnetkette der senkrechte Abstand zwischen inzisal-oraler Kante und labialer Kante des Drahtes im Mittel 0,04950 mm kleiner ist. Neben der verwendeten Methode konnte auch bei der Lokalisation des Retainerdrahtes ein signifikanter Einfluss (p-Wert $<0,0001)$ nachgewiesen werden. Der Minimalwert des senkrechten Abstandes in Gruppe IrregularityIndex nach Little 0-1 lag an Zahn 33 mit einem Mittelwert von 0,326 mm (95\%Konfidenzintervall 0,287 mm - 0,388 mm) unter Verwendung der Neodym-Magnetkette. Der Maximalwert für diese Modellgruppe lag mit einem Mittelwert von 0,502 mm (95\%Konfidenzintervall 0,379 mm - 0,481 mm) an Zahn 32 unter Verwendung der Einsatzhilfe.

Auch in der Modellgruppe Irregularity-Index nach Little 4-10 ließ sich ein signifikanter Einfluss der Methode auf den senkrechten Abstand (p-Wert 0,0074) nachweisen. Allerdings liegt eine qualitative Wechselwirkung zwischen Gruppe und Methode vor, das heißt, dass nun mit der Standardmethode die geringeren Distanzen erzielt werden. Der Unterschied wird auf 0,0432 mm (95\%-Konfidenzintervall -0,07548 mm - -0,01110 mm) geschätzt.

Neben der angewendeten Methode ließ sich auch in der Modellgruppe Irregularity-Index nach Little 4-10 bei der Lokalisation ( $p$-Wert $<0,0001$ ) ein signifikanter Einfluss feststellen.

Der Minimalwert des senkrechten Abstandes in dieser Modellgruppe lag im Mittel an Zahn 33 mit einem Wert von 0,296 mm (95\%-Konfidenzintervall 0,246 mm - 0,347 mm), nun jedoch unter Verwendung der Einsatzhilfe. Der Maximalwert des senkrechten Abstandes wurde unter Verwendung der Neodym-Magnetkette mit einem Mittelwert von 0,525 mm (95\%-Konfidenzintervall 0,474 mm - 0,575 mm) an Zahn 32 gemessen.

Abbildung 12 stellt die Messwerte der Distanz [mm] von der Zahnoberfläche der Zähne 33, 32, 31, 41, 42, 43 zur Drahtoberfläche grafisch dar, ohne dabei den Drahttyp zu berücksichtigen. 
95\% Konfidenzintervalle der Distanz

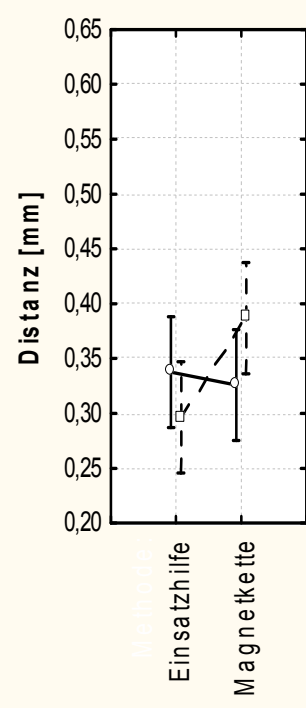

Zahn 33

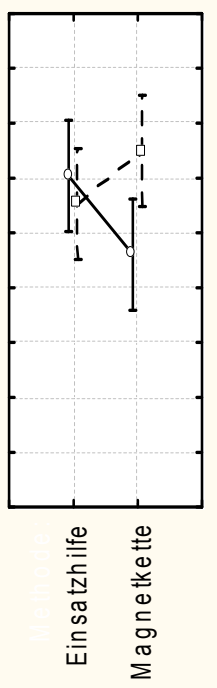

Zahn 32

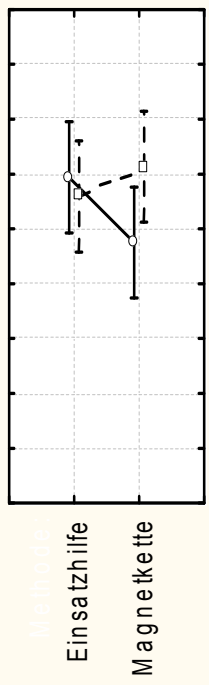

Zahn 31

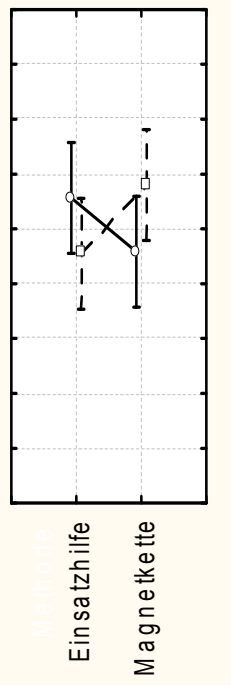

Zahn 41

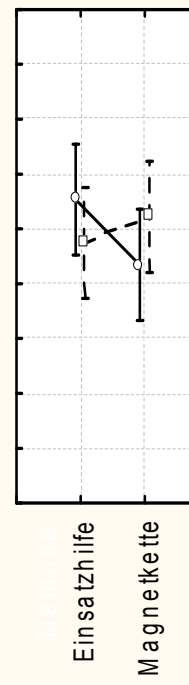

Zahn 42

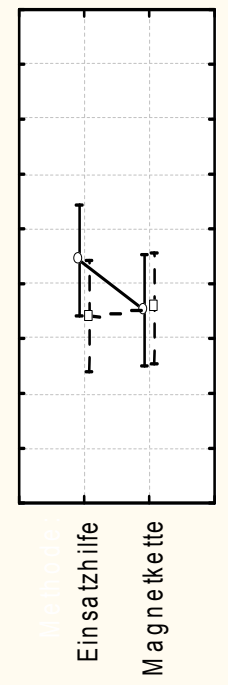

Zahn 43

Abbildung 12: Zusammenfassung aller Messwerte des senkrechten Abstandes in [mm] von der inzisal-oralen Kante der Zähne 33, 32, 31, 41, 42, 43 zur labialen Begrenzung des Retainerdrahtes im Mittelwert unabhängig von der Drahtstärke. Verglichen wurde die Größe der Distanz bei Verwendung der Standardmethode mit Einsatzhilfe und der neuen Methode mit Magnetkette. Die Methoden wurden jeweils an 40 Unterkiefermodellen mit einem Index nach Little von 0-1 (keine bis geringe Irregularität) und einem Index nach Little von 4-10 (moderate bis starke Irregularität) untersucht. durchgängige Linie = Index nach Little 0-1 gestrichelte Linie = Index nach Little 4-10

\subsection{Auswertung der Biegezeit}

Wie in Abbildung 13 grafisch dargestellt, beträgt die Biegezeit [min] für die Verwendung eines 15er Twistflex (Dentaurum, Ispingen, Deutschland) bei den Modellen mit einem Index nach Little von 0-1 im Mittel 11,12 min (95\%-Konfidenzintervall 10,52 min - 11,73min) unter Verwendung der Standardmethode mit Einsatzhilfe. Bei Verwendung der NeodymMagnetkette beträgt der Mittelwert der Biegezeit eines Retainers aus 15er Twistflex 9,33 min (95\%-Konfidenzintervall Minimum 8,73 min, Maximum 9,93 min).

Die Biegezeit beträgt bei Modellen mit einem Index nach Little 4-10 unter Verwendung der gleichen Drahtstärke im Mittelwert 11,99 min (95\%-Konfidenzintervall Minimum 11,39 min, Maximum 12,59 min) für die Methode mit Einsatzhilfe und 10,07min (95\%- 
Konfidenzintervall Minimum 9,48 min, Maximum 10,68 min) für die Methode mit der Neodym-Magnetkette.

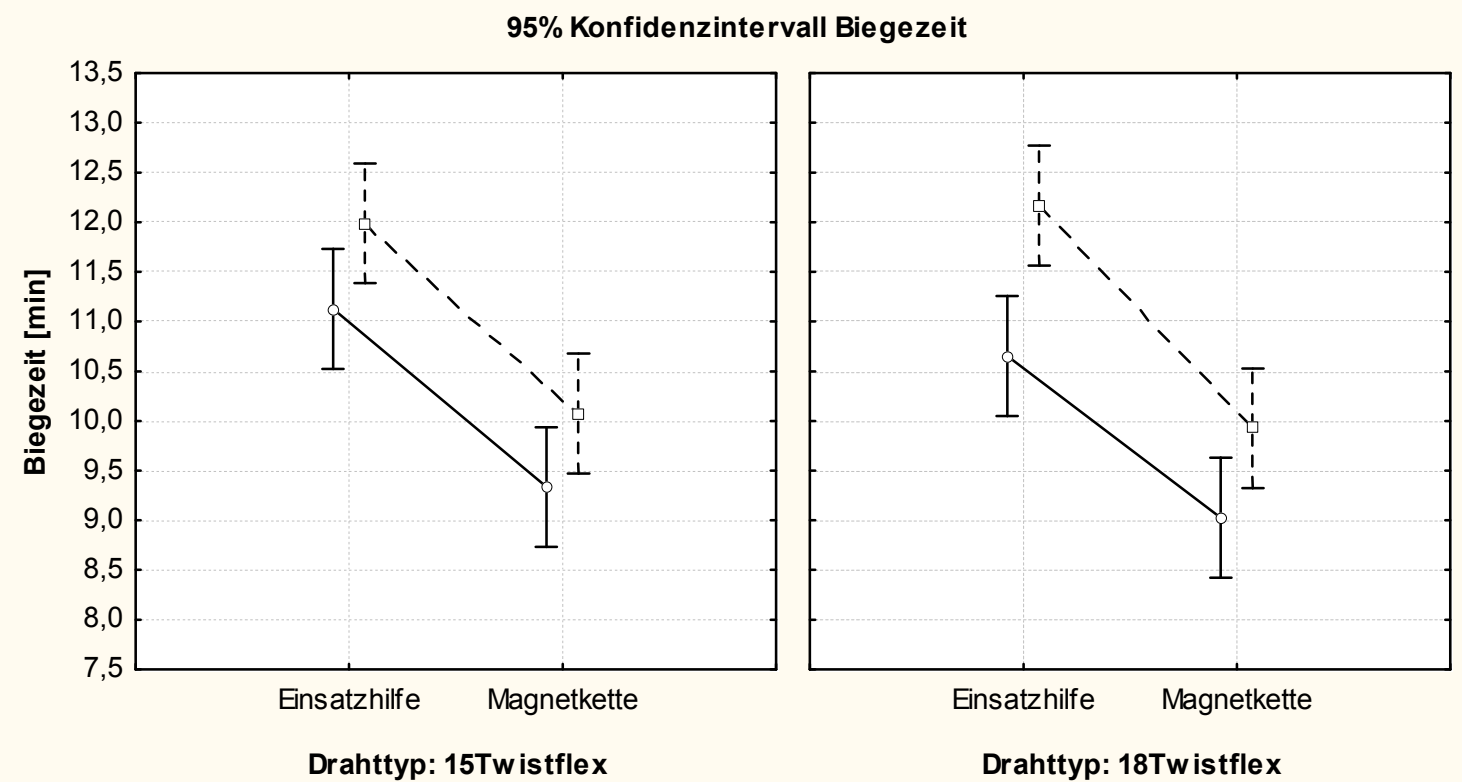

Abbildung 13: Dargestellt wird die Biegezeit [min] eines Zahntechnikers der Universitätsmedizin Göttingen bei Verwendung eines 15er Twistflex-Drahtes und eines 18er Twistflex-Drahtes. Verglichen werden die Standardmethode mit Einsatzhilfe und die neue Methode mit Magnetkette. Die Methoden wurden jeweils an 20 Unterkiefermodellen mit einem Index nach Little von 01 (keine bis geringe Irregularität) und einem Index nach Little von 4-10 (moderate bis starke Irregularität) untersucht. durchgängige Linie = Index nach Little 0-1 gestrichelte Linie = Index nach Little 4-10

Bei Verwendung des 18er Twistflex ergab sich für die Gruppe Index nach Little 0-1 bei der Standardmethode eine Biegezeit von 10,65 min (Minimum 10,05 min, Maximum 11,26 min) und bei der Methode mit Magnetkette eine Zeit von 9,03 min (Minimum 8,42 min, Maximum 9,63 min). Für die Gruppe Index nach Little 4-10 ergab sich bei der Standardmethode eine Biegezeit von 12,17 min (Minimum 11,56 min, Maximum 12,77 min) im Mittel und für die Magnetkette eine Biegezeit von 9,93 min (Minimum 9,32 min, Maximum 10,53 min) im Mittel.

Es lässt sich demnach ein signifikanter Einfluss der Methode auf die Biegezeit nachweisen (pWert $<0,0001)$. Der Unterschied in der Biegezeit wird auf 1,89 min geschätzt, d.h. im Mittel 
ist die Methode mit der Magnetkette um 1,89 min schneller. Es lässt sich ebenfalls ein signifikanter Einfluss der Gruppe nach dem Irregularitätsindex nach Little auf die Biegezeit nachweisen ( $\mathrm{p}$-Wert < 0,0001). Der Herstellungsprozess eines Retainers für die Modelle Gruppe Index nach Little 4-10 dauerte für beide Drahttypen und beide Herstellungsmethoden signifikant länger. Der Drahttyp hat keinen signifikanten Einfluss auf die Biegezeit (p-Wert = $0,3864)$.

\subsection{Auswertung der Befestigungszeit}

Das Befestigen eines 3-3-Retainers auf dem jeweiligen Gipsmodell durch einen Facharzt der Abteilung für Kieferorthopädie der Universitätsmedizin Göttingen wurde in [min] gemessen. In Abbildung 14 wird die Befestigungszeit unter Berücksichtigung der jeweiligen Befestigungsmethode, der unterschiedlichen Drahtstärken sowie der unterschiedlichen Modellgruppen grafisch dargestellt.

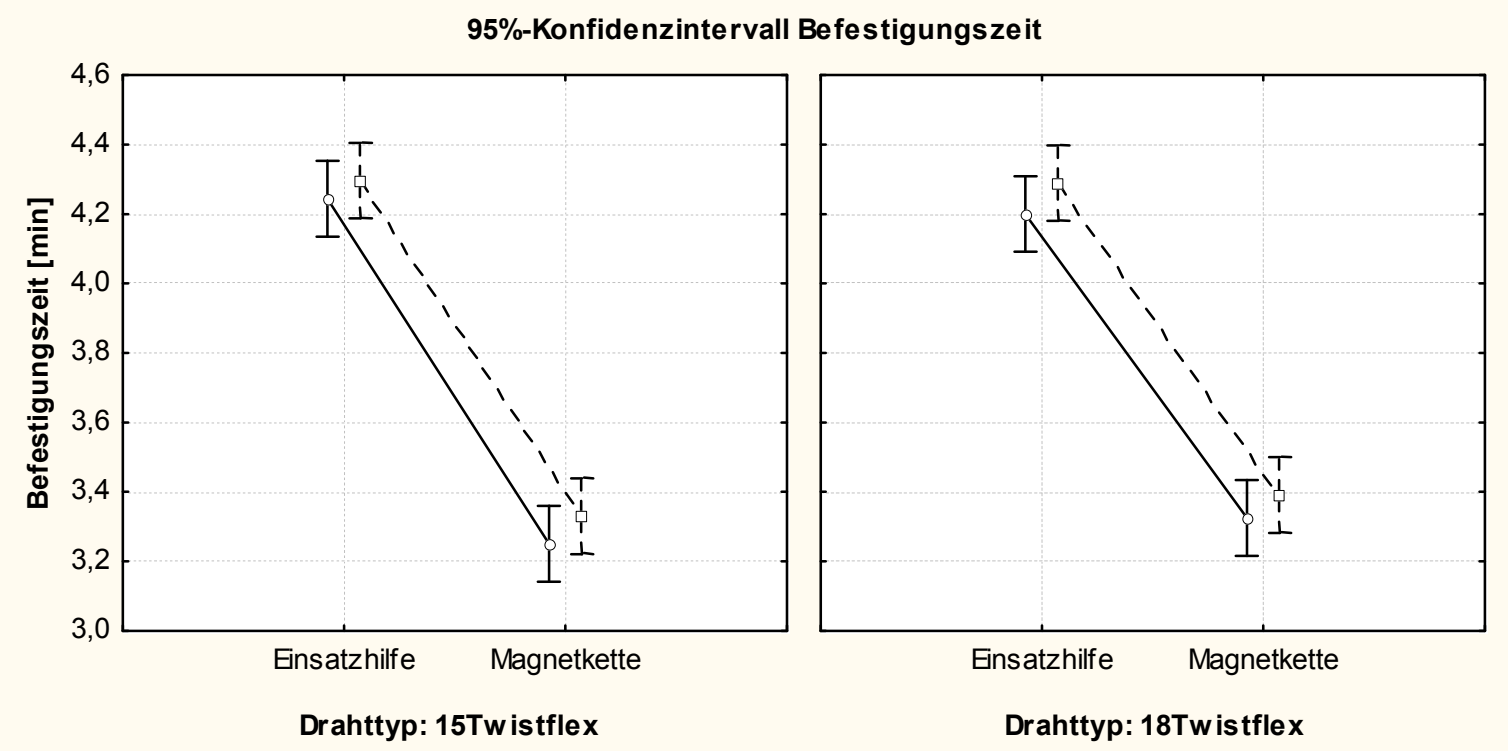

Abbildung 14: Dargestellt wird die Befestigungszeit [min] eines 3-3-Retainers aus einem 15er oder 18er Twistflex-Drahtes durch einen Facharzt der Abteilung für Kieferorthopädie der Universitätsmedizin Göttingen. Verglichen werden die Standardmethode mit Einsatzhilfe und die neue Methode mit Magnetkette. Die Methoden wurden jeweils an 20 Unterkiefermodellen mit einem Index nach Little von 0-1 (keine bis geringe Irregularität) und einem Index nach Little von 4-10 (moderate bis starke Irregularität) durchgeführt.

durchgängige Linie = Index nach Little 0-1

gestrichelte Linie = Index nach Little 4-10 
Der Mittelwert der Befestigungszeit eines 3-3 Retainers aus einem 15er Twistflex liegt bei der Gruppe mit einem Index nach Little von 0-1 unter Verwendung der Standardmethode bei 4,3 min (Minimum 4,13 min, Maximum 4,34 min) und bei der Magnetkette bei 3,25 min ( 3,13 min, Maximum 3,36 min]). Bei der Befestigung eines Retainers der gleichen Drahtstärke bei Modellen der Gruppe Index nach Little 4-10 ergab sich bei Verwendung der Methode mit Einsatzhilfe ein Mittelwert der Befestigungszeit von 4,3 min (Minimum 4,19 min, Maximum 4,40 min). Bei Verwendung der Neodym-Magnetkette für die adhäsive Befestigung konnte ein Mittelwert von 3,33 min (Minimum 3,21 min, Maximum 3,44 min) ermittelt werden.

Der Mittelwert für die Befestigungszeit eines Retainers aus 18er Twistflex für Modelle der Gruppe Index nach Little $0-1$ bei Verwendung der Standardmethode beträgt 4,2 min (Minimum 4,1 min, Maximum 4,31 min). Bei der Methode mit der Neodym-Magnetkette liegt der Mittelwert bei dieser Gruppe bei 3,33 min (Minimum 3,22 min, Maximum 3,42 $\min )$.

Für die Gruppe Index nach Little 4-10 wurde eine Befestigungszeit für einen Retainer aus 18er Twistflex bei Durchführung der Standardmethode mit einem Mittelwert von 4,29 min (Minimum 4,20 min, Maximum 4,38 min) ermittelt. Mit der Magnetkette konnte ein Mittelwert für die Befestigungszeit von 3,38 min (Minimum 3,27 min, Maximum 3,50 min) erreicht werden.

Die verwendete Methode hat einen signifikanten Einfluss auf die Befestigungszeit (p-Wert $<0,0001)$. Der Unterschied zwischen den Methoden Einsatzhilfe vs. Magnetkette wird hierbei auf 0,93 min (Minimum 0,85 min, Maximum 1,01 min) geschätzt. Im Mittel ist die Methode mit Magnetkette zur Befestigung eines 3-3-Retainers um 0,93 min schneller. Ein signifikanter Einfluss des Drahttyps auf die Befestigungszeit ist nicht zu erkennen ( $\mathrm{p}$-Wert $=0,5887)$. 


\section{$5 \quad$ Diskussion}

Nach Abschluss einer aktiven kieferorthopädischen Behandlung werden häufig 6-BasenRetainer zur Prophylaxe eines tertiären Engstandes eingesetzt.

Zur Verringerung von Scherbelastungen auf den Retainer sollte dieser möglichst passgenau gefertigt und befestigt sein. Scherbelastungen treten vor allem beim Abbeißen und Kauen von fester Nahrung auf. Aber auch die Auslenkung der Zähne z.B. durch Parafunktionen kann zu starken Spannungen zwischen Retainerdraht und Kunststoff führen. Durch diese Belastungen können adhäsive und kohäsive Brüche entstehen (Radlanski und Zain 2004). Als adhäsiven Bruch bezeichnet man das Ablösen der Schmelz-Komposit-Verbindung. Der Bruch der Verbundstelle von Draht und Komposit wird als kohäsiver Bruch bezeichnet. Vor allem Fehler im adhäsiven Befestigungsprozess des Drahtes begünstigen diese Misserfolge und den daraus resultierenden Retentionsverlust (Lumsden et al. 1999, Segner und Heinrici 2000). In der Literatur werden eine Reihe von Voraussetzungen beschrieben, die zur Vermeidung dieser Fehler beitragen können (Bryan und Sherriff 1995, Dahl und Zachrisson 1991, Radlanski und Zain 2004).

Zunächst sollte der Retainer den Zahnoberflächen möglichst exakt anliegen, damit er weniger Scherbelastungen ausgesetzt ist. In der vorliegenden Studie wurde die Passgenauigkeit adhäsiv geklebter 3-3-Retainer aus einem 3-fach verseiltem Twistflex der Stärken 0,38 mm (15er Dentaflex; Dentaurum, Ispingen, Deutschland) und 0,45 mm (18er Dentaflex; Dentaurum, Ispingen, Deutschland) untersucht. Verglichen wurden die temporäre Fixierung und Positionierung der Retainerdrähte mit einer durch einen Zahntechniker hergestellten Einsatzhilfe aus Kunststoff und mit einer aus Neodym-Magneten hergestellten Kette. Des Weiteren wurde der Einfluss der Drahttypen auf die Passung der Retainer sowie die Dauer der Herstellungs- und Befestigungsprozesse untersucht.

Die Ergebnisse zeigten, dass bei Verwendung der Magnetkette die Passung der Retainer aus beiden Drahtstärken geringfügig besser war als bei Verwendung der Einsatzhilfe. Die statistische Auswertung zeigte einen Unterschied der Fläche zwischen der lingualen Zahnoberfläche und der labialen Kante des Drahtbogens bei Verwendung der aus Kunststoff gefertigten Einsatzhilfe und der Magnetkette von $0,8234 \mathrm{~mm}^{2}$. Die Fläche war bei der Befestigung des Retainerdrahtes mit der Magnetkette signifikant kleiner ( $\mathrm{p}$-Wert = 0,0347). Der Irregularitätsindex nach Little spielte bei der Auswertung der Fläche $\left[\mathrm{mm}^{2}\right]$ keine signifikante Rolle. Um die Passgenauigkeit des Retainerdrahtes an den einzelnen Zähnen zu untersuchen, wurde außerdem der senkrechte Einzelabstand [mm] der Zahnoberfläche zur labialen Begrenzung des Retainerdrahtes gemessen. Die Ergebnisse zeigten, dass auch hier 
die Differenzen bei Verwendung der unterschiedlichen Methoden sehr gering waren. Die verwendete Drahtstärke hatte keinen signifikanten Einfluss auf die erzielten Ergebnisse. In der Modellgruppe mit einem Irregularitätsindex nach Little von 0-1 ist die Distanz der einzelnen Messpunkte bei Verwendung der Magnetkette signifikant kleiner als bei Verwendung der Einsatzhilfe $(\mathrm{p}$-Wert $=0,0002)$. Der Unterschied liegt bei 0,04950 mm. Wurden die Retainer in der Modellgruppe Irregularitätsindex nach Little 4-10 mit der konventionellen Einsatzhilfe positioniert und geklebt, so konnte man mit dieser Methode geringere Distanzen [mm] zwischen Zahnoberfläche und labialer Drahtkante erreichen (pWert $=0,0074)$. Der Unterschied wird hier auf -0,0432 mm geschätzt.

Während der Positionierung des Retainers mit der Magnetkette wurde dieser nur durch die Magnetkraft gehalten und konnte in kleinerem Ausmaß frei auf den Zahnoberflächen verschoben werden. Es war somit keine reproduzierbare, vom Zahntechniker vorgegebene Idealposition einstellbar. Bei der Verwendung einer Einsatzhilfe legt der Zahntechniker die von ihm als ideal angenommene Position des Drahtes fest. Dennoch wurde mit Hilfe der Magnetkette eine geringfügig bessere Passung des Retainers erzielt. Dies lässt sich wahrscheinlich durch eine Selbstpositionierung des Drahtes im Wechselspiel zwischen Magnetkraft, Zahnkonturen und Draht erklären. Wird der Draht durch den Behandler an den Zähnen ausgerichtet, kommt es zu einem Rutschen und Gleiten des Drahtes in die bestmögliche Passung. Durch die gleichmäßig wirkenden Magnetkräfte auf dem lingual konvex geformten Zahnbogen rutscht der Draht in die beste Passungsposition. So kommen zwangsläufig die meisten Drahtbogenanteile mit der Zahnoberfläche in Kontakt.

In der Gruppe Irregularitätsindex 4-10, was einer moderaten bis starken Irregularität des Zahnbogens entspricht, ist dieses Phänomen der Selbstjustierung des Drahtes nur eingeschränkt möglich. Durch den irregulär geformten Zahnbogen ist das Gleiten des Drahtes in seine Idealposition nicht optimal möglich. Dadurch lässt sich erklären, warum in dieser Gruppe die Einsatzhilfe eine geringfügig bessere Passung erreichen konnte.

Um die Rate an kohäsiven Brüchen, also Brüchen zwischen Komposit und Draht gering zu halten, ist es wichtig, dass der Draht während des adhäsiven Befestigens spannungsfrei anliegt. Starkes Anpressen des Drahtes an die Zahnoberfläche während des Klebevorgangs kann zu Spannungen zwischen Draht und Komposit führen. Diese kann bei Auslenkung der Zähne unter Last über einen Schwellenwert gesteigert werden und zu einem Bruch der Klebestelle führen. Mit Hilfe der Magnetkette liegt der Retainer während des adhäsiven Befestigungsvorgangs den lingualen Zahnoberflächen gleichmäßig passiv an. Die Magnetkräfte sind dabei so gering, dass es nicht zu einer Verformung des Drahtes kommt. Da 
durch die Magnetkette die lingualen Flächen vollständig zugänglich sind und somit gleichzeitig mit Komposit bestückt werden können, ist ein nachträgliches Andrücken des Drahtes an einer Klebestelle nicht mehr notwendig.

Verschiedene Autoren beschreiben zahlreiche Positionierhilfen, die den Retainer während des adhäsiven Befestigungsvorgangs auf die Zahnoberfläche drücken. Eade (1980) beschreibt eine Technik, bei der mit Hilfe von autopolymerisierendem Kunststoff Übertragungskappen am Retainer befestigt werden, welche an den Eckzähnen lokalisiert sind. Nach abgeschlossenem Klebevorgang werden diese Kappen entfernt. Hobson und Eastough (1993), Zekic und Gelgör (2004) und Acharya et al. (2004) beschreiben Methoden zur Erleichterung des Klebevorganges mit Übertragungshilfen aus Silikon oder Kunststoff. Hierbei ist es häufig nicht sicher zu kontrollieren, ob der Draht wirklich spannungsfrei anliegt. Aus diesem Grund ist es sehr wahrscheinlich, dass ein 6-Basen-Retainer mit Hilfe einer Magnetkette spannungsfreier als mit Hilfe einer laborgefertigten Positionierhilfe adhäsiv befestigt werden kann. In einer folgenden Studie soll geklärt werden, ob die bessere Passung sowie die spannungsfreiere adhäsive Befestigung der mit Hilfe der Magnetkette hergestellten Retainer bei diesen auch zu einer höheren Überlebensrate führen.

Um die Gefahr eines adhäsiven Bruchs, also das Ablösen des Kunststoffes vom Schmelz, zu minimieren, ist ein zügiger und überschaubarer Befestigungsvorgang des Drahtes notwendig. Durch einen schnellen Befestigungsprozess kann auch die Kontamination der geätzten Schmelzoberfläche mit Speichel, Sulkusflüssigkeit, Feuchtigkeit aus der Atemluft und Blut vermieden werden (Axellson und Zachrisson 1992).

Bereits eine klinische Studie an 45 Patienten (Hahn et al. 2008) konnte zeigen, dass die Retainer mit Hilfe der Magnetkette signifikant schneller befestigt werden konnten als unter Verwendung von Zahnseide und einer individuell gefertigten Einsatzhilfe aus Kunststoff, wie sie auch in dieser Studie verwendet wurde. Dieses Ergebnis deckt sich mit den Ergebnissen der vorliegenden Studie. Die statistische Auswertung konnte einen signifikanten Einfluss der Methode auf die Befestigungszeit nachweisen ( $p$-Wert $<0,0001$ ). Die Befestigungszeit der Retainer mit Hilfe der Magnetkette war um 0,93 Minuten schneller als die Befestigungszeit mit Hilfe der konventionellen Methode. Die reduzierte Zeit für die adhäsive Befestigung verringert die Wahrscheinlichkeit der Kontamination der geätzten Schmelzoberfläche mit Speichel, Sulkusflüssigkeit oder Blut, was die Haltbarkeit der Retainer nachteilig beeinflussen kann (Andrén et al. 1998, Dahl und Zachrisson 1991). Durch die labiale Befestigung der Magnetkette ist das lingual gelegene Arbeitsfeld übersichtlicher, und eine einfachere und schnellere Trockenlegung wird ermöglicht. Bei der aus Kunststoff gefertigten Einsatzhilfe, 
aber auch bei den aus Zahnseide gefertigten lingual gelegenen Schlaufen, ist eine erhöhte Vorsicht während des adhäsiven Befestigens des Retainers für den Behandler geboten. Durch die lingual liegenden Anteile dieser temporären Fixiertechniken besteht die Gefahr, dass sich das Adhäsiv mit der Übertragungshilfe oder der Zahnseide verbindet und ein späteres Entfernen erschwert wird. Aufgrund dieser Problematik wird eine erhöhte Konzentration des Behandlers gefordert, und diese bedingt ein langsameres Arbeitstempo. Wird ein Retainer mit Hilfe der konventionellen Einsatzhilfe adhäsiv geklebt, so muss der Arbeitsvorgang des Klebens aufgeteilt werden. Zunächst werden die distalen Enden des Retainers adhäsiv befestigt, anschließend wird die Einsatzhilfe entfernt und abschließend die Basen in Region der Inzisivi geklebt. Dieser geteilte Arbeitsgang erfordert mehr Zeit als beim Befestigen des Drahtes mit Hilfe der Magnetkette. Bei deren Einsatz können alle 6 Basen in einem Arbeitsschritt geklebt werden. Abschließend wird die in Wachs gebettete Magnetkette durch einfaches Abziehen entfernt. Das Entfernen der Einsatzhilfe erhöht zusätzlich die Gefahr eines kohäsiven Bruchs, da durch das Entfernen des Kunststoffes vom Draht dieser erneut unter Spannung gesetzt wird.

Auch die Ergebnisse der Herstellungszeit der Retainer durch den Zahntechniker zeigten, dass mit Hilfe der Magnetkette die Retainerherstellung signifikant schneller möglich ist (p-Wert < 0,0001). Der Unterschied in der Herstellungszeit wird auf 1,89 Minuten geschätzt. Die schnellere Herstellungszeit lässt sich vor allem durch das Einsparen eines Arbeitsschrittes erklären. Die Magnetkette ist vorgefertigt und muss bei der Herstellung nur mit Wachs auf dem Arbeitsmodell fixiert werden. Bei der Herstellung eines Retainers mit Einsatzhilfe muss diese nach Anpassen des Retainers an die linguale Zahnoberfläche hergestellt werden, wodurch sich der Herstellungsprozess verlängert. Des Weiteren wird der Herstellungsprozess für den Zahntechniker durch die Magnetkette vereinfacht. Durch die gleichmäßig wirkenden magnetischen Kräfte wird der Draht in Position gehalten, und der Zahntechniker muss zur Kontrolle der Passgenauigkeit den Draht nicht immer wieder erneut an das Modell anlegen. Dadurch kann der Herstellungsprozess zusätzlich verkürzt werden.

Zusätzlich wurde der mögliche Einfluss unterschiedlicher Drahtqualitäten auf die Passgenauigkeit sowie auf die Dauer des Herstellung- und Befestigungsprozesses eines Retainers bei Verwendung der beiden in dieser Studie verwendeten temporären Fixiertechniken untersucht. Um diese Fragestellung zu klären wurden in dieser Studie 3-fach verseilten Drähte (15 Dentaflex und 18 Dentaflex, Dentaurum, Ispingen, Deutschland) der Stärken 0,38 mm (0,015 inch) und 0,45 mm (0,018 inch) verwendet. Bei diesen Drahttypen handelt es sich um die für die Herstellung von Retainern gängigen Stärken. Drähte mit einer 
Stärke von $0,38 \mathrm{~mm}$ werden vorwiegend bei parodontalen Risikofällen eingesetzt, da sie die physiologische Zahnbeweglichkeit am wenigsten einschränken (Schwarze et al. 1995). Retainerdrähte mit einer Stärke von $0,45 \mathrm{~mm}$ finden allgemein bei der Herstellung von Drahtbogenretainern Anwendung. Andrén et al. 1998 konnten in einer Langzeitstudie zeigen, dass Retainer dieser Drahtstärke eine geringere Verlustrate aufweisen als Retainer mit einer Stärke von 0,38 mm. Die statistische Auswertung ergab, dass beide untersuchten Drahttypen keinen signifikanten Einfluss auf die Ergebnisse der Passung hatten. Ebenso war der Einfluss der unterschiedlichen Drahttypen auf die Herstellungs- und Befestigungszeiten eines Retainers nicht signifikant. Diese Ergebnisse zeigen, dass die Auswahl der Befestigungsmethode unabhängig von dem ausgewählten Drahttyp ist.

Hervorzuheben ist, dass die gemessenen sehr geringen Unterschiede bezüglich der Passgenauigkeit zwischen beiden Verfahren für sich genommen nicht für die klinische Überlegenheit der Magnetkette sprechen. Die leicht bessere Passung hat gezeigt, dass die fehlende Positionierung bei Verwendung der Magnetkette letztendlich kein Problem darstellt und die Vorteile der schnelleren Befestigungs- sowie Herstellungszeit nicht mit einer geringeren Passung einhergehen. Bei Verwendung der Magnetkette ist jedoch mit geringeren Kosten zu rechnen, da verschiedene Laborschritte zur Anfertigung einer Einsatzhilfe entfallen. Zudem lassen sich der Herstellungsprozess und der klinische Befestigungsprozess zeitlich optimieren, was auch positiv auf den Kostenfaktor einwirkt.

NdFeB-Magnete finden nicht nur in der Industrie und Technik Anwendung, sondern wurden auch schon mehrfach in der Medizin und Zahnheilkunde angewendet. So wurden Magnete zur Therapie retinierter oberer Canini (Sandler 1991, Darendeliler und Friedle 1994), zur Retention (Springate und Sandler 1991), zur Korrektur des offenen Bisses (Dellinger 1986) und in funktionskieferorthopädischen Apparaturen (Vardimon et al. 1989, 1990, Darendeliler und Joho 1993, Darendeliler et al. 1993) verwendet. NdFeB-Magnete zeigen eine hohe magnetische Kraft im Vergleich zu ihrer kleinen Größe und haben eine sehr hohe Resistenz gegen Entmagnetisierung (Noar und Evans 1999).

Nachteil dieser Magnete ist ihre Korrosionsanfälligkeit in unbeschichtetem Zustand (Wilson et al. 1995, Wilson et al. 1997). Eine Studie von Wilson et al. (1995) zeigte jedoch, dass $\mathrm{Nd}_{2} \mathrm{Fe}_{14} \mathrm{~B}$-Magnete mit einer Beschichtung aus Parylene keine Korrosion zeigen. Bei Verwendung der Magnetkette am Patienten ist es möglich, diese mit Hilfe eines Thermodesinfektors zu desinfizieren ohne dass sie dadurch Schaden nimmt. Eine Sterilisation ist aufgrund der geringen Hitzebeständigkeit der Kunststoffummantelung nicht möglich. 
$\mathrm{Nd}_{2} \mathrm{Fe}_{14} \mathrm{~B}-$ Magnete sollten nicht bei Patienten mit Herzschrittmachern verwendet werden ( $\mathrm{Li}$ 2007, Wolber et al. 2007). Durch das magnetische Feld kann der Pulsgenerator des Herzschrittmachers in einen Magnetmodus geschaltet werden. In diesem Magnetmodus wird das Herz nicht mehr im Rhythmus des Patienten stimuliert, sondern in einer festen Frequenz. Folge kann ein Flimmern sein, bei dem die Herzbewegung unkoordiniert abläuft und nicht mehr genügend Blut in den Kreislauf gepumpt wird.

Auch die auf die Verwendung von $\mathrm{Nd}_{2} \mathrm{Fe}_{14} \mathrm{~B}$-Magneten in der Nähe von magnetisierbaren Speichermedien sollte vermieden werden (Hahn et al. 2008).

\subsection{Schlussfolgerung}

Mit Hilfe einer NeFeB-Magnetkette zur temporären Fixierung von 6-Basen-Retainern während des adhäsiven Befestigungsvorgangs ist trotz Fehlens einer exakten Positionierung des Drahtes in der vom Zahntechniker als ideal erachteten Position eine etwas bessere Passung im Vergleich zur Fixierung mit einer individuell aus Kunststoff gefertigten Einsatzhilfe zu erzielen. Durch das gleichmäßig wirkende magnetische Feld der NeFeBMagnetkette wird der Draht, der in seiner Zuordnung zu den Labialflächen der Zähne frei ist, in eine Position mit bestmöglicher Passung gezogen, ohne dadurch verformt zu werden. Durch diese Gegebenheiten während des adhäsiven Befestigungsprozesses kann von einem passiven Sitz des Retainers und einem relativ spannungsfreien Verbund zwischen Draht und Komposit ausgegangen werden. In einer nachfolgenden Studie soll geklärt werden, ob dies auch zu einer höheren Überlebensrate des Retainers führt.

Der Einsatz der NeFeB-Magnetkette bei der zahntechnischen Herstellung eines 6-BasenRetainers, sowie bei der adhäsiven Befestigung des Retainers beim Patienten, konnte diese Prozesse zeitlich deutlich optimieren und die Prozesse für den Zahntechniker sowie den Behandler vereinfachen. Da die Magnetkette vorgefertigt und mehrfach verwendbar ist, entfällt für den Zahntechniker die Herstellung der individuellen Einsatzhilfe, was neben der Zeitersparnis auch den Kostenfaktor günstig beeinflusst. Durch den labialen Sitz der Magnetkette im Mund des Patienten werden ein übersichtlicherer Klebevorgang sowie eine schnellere Trockenlegung ermöglicht. Diese Faktoren können sich auch positiv auf die Überlebensrate des Retainers auswirken, da die Wahrscheinlichkeit der Kontamination der geätzten Schmelzoberfläche mit Speichel, Sulkusflüssigkeit oder Blut reduziert wird. Diese These soll jedoch durch eine folgende klinische Langzeitstudie überprüft werden.

Die Dauer der Herstellung sowie der Befestigung von Retainern mit konventioneller Einsatzhilfe und Magnetkette wird durch unterschiedliche Drahttypen nicht beeinflusst. Auch 
haben die unterschiedlichen Drahtstärken keinen Einfluss auf die Passgenauigkeit der mit in dieser Studie untersuchten Fixiertechniken hergestellten und adhäsiv befestigten Retainer. Mit beiden temporären Fixiertechniken können Retainerdrähte unterschiedlicher Stärke ohne Nachteile hinsichtlich der Anwendungdauer und der anschließenden Passgenauigkeit der Retainer verwendet werden.

Zusammenfassend kann festgestellt werden, dass mit der Neodym-Eisen-Bor-Magnetkette eine Methode zur Verfügung steht, mit deren Hilfe Drahtbogenretainer unterschiedlicher Drahtstärken nach Abschluss einer KFO-Behandlung schneller und passgenauer befestigt werden können. Aus theoretischen Überlegungen lässt sich herleiten, dass auch eine spannungsfreiere Befestigung gegenüber der Verwendung von konventionellen Positionierhilfen möglich ist. $\mathrm{Ob}$ die gezeigten Verbesserungen durch das neue Verfahren auch einen positiven Einfluss auf die langfristige Überlebensrate von 3-3-Retainern haben, soll Gegenstand einer klinischen Langzeitstudie sein. 


\section{$7 \quad$ Zusammenfassung}

Seit der Entwicklung lingual geklebter 3-3-Retainer wurden verschiedene Techniken entwickelt, um den Klebevorgang am Patienten zu optimieren. In dieser Studie wurden eine konventionelle Methode mit laborgefertigter Einsatzhilfe und eine neu entwickelte Methode mit einer Neodym-Magnetkette zur temporären Fixation von 3-3-Retainern während der Herstellung und Befestigung verglichen. Als Parameter dienten die Dauer der Herstellung und Befestigung sowie die Passgenauigkeit der Retainer. Zusätzlich wurde der Einfluss unterschiedlicher Drahttypen auf die Dauer der Herstellungs- und Befestigungszeit sowie auf die Passung der Retainer untersucht.

Nach Herstellung und Vervielfältigung von Gipsmodellen des Unterkiefers und Einteilung in zwei Gruppen (Irregularity-Index nach Little 0-1 und 4-10) wurden die 3-3-Retainer durch einen Zahntechniker der Universitätsmedizin Göttingen mit Hilfe der unterschiedlichen Herstellungsmethoden und aus zwei verschiedenen Drahtqualitäten (15er und 18er Dentaflex) gefertigt. Die Dauer des Herstellungsprozesses wurde gemessen und dokumentiert. Die Untersuchungsmodelle wurden in klinische Simulationseinheiten montiert. Danach erfolgte die adhäsive Befestigung der 3-3-Retainer mit der konventionellen Einsatzhilfe und der Neodym-Magnetkette durch einen Fachzahnarzt für Kieferorthopädie. Die benötigte Zeit für die adhäsive Befestigung wurde gemessen und dokumentiert. Anschließend wurde aus allen Modellen das Front-Eckzahnsegment herausgetrennt und dieses in der Vertikalen bis zum Retainerdraht mit einem Feintrimmer reduziert. Jedes so bearbeitete Modell wurde eingescannt und jeweils die Flächen und senkrechten Abstände zwischen den Zähnen und dem Retainer vermessen.

Es konnte gezeigt werden, dass der Herstellungsvorgang mit Hilfe der Neodym-Magnetkette um 1,89 Minuten signifikant schneller als mit der Einsatzhilfe war. Der Klebevorgang bei Verwendung der Magnetkette war um 0,93 min schneller als bei Verwendung der Einsatzhilfe. Die statistische Auswertung der Fläche zwischen Drahtbogen und Zahnbogen ergab, dass diese bei den mit der Magnetkette geklebten Retainern in beiden Gruppen signifikant kleiner war. Der Drahttyp hatte keinen signifikanten Einfluss auf die Herstellungsund Befestigunszeit sowie auf die Passung der Retainer.

Abschließend ließ sich feststellen, dass mit Hilfe der Neodym-Magnetkette der Herstellungsund adhäsive Befestigungsvorgang von lingual geklebten 3-3-Retainern zeitlich optimiert werden konnte. Die Passgenauigkeit der mit der Neodym-Magnetkette geklebten Retainer war in Bezug auf die Fläche zwischen Drahtbogen und Zahnbogen der Passgenauigkeit konventionell geklebter Retainer etwas überlegen. Außerdem lässt sich aus ergonomischer 
Sicht sagen, dass die Magnetkette ein einfacheres, übersichtlicheres und kostengünstigeres Arbeiten für den behandelnden Zahnarzt erlaubt, als dies mit herkömmlichen Positionierungshilfen möglich ist. 


\section{$7 \quad$ Literaturverzeichnis}

Acharya B, Acharya D, Keluskar KM (2004): A simple transfer tray for bonding lingual retainers. J Clin Orthod $\underline{38}, 557-9$

Al-Emran S, Barakati R (2007): A method of stabilizing a lingual fixed retainer in place prior to bonding. J Contemp Dent Pract $\underline{8(7)}, 1-5$

Andrén A, Asplund J, Azarmidohkt E, Svensson R, Varde P, Mohlin B (1998): A clinical evaluation of long term retention with bonded retainers made from multi-strand wires. Swed Dent J 22, 123-131

Angle EA: Treatment of malocclusion of the teeth. $7^{\text {th }}$ ed. SS White Manufacturing Co., Philadelphia 1907

Årtun J (1984): Caries and periodontal reactions associated with long-term use of different types of bonded lingual retainers. Am J Orthod $\underline{86}, 112-8$

Årtun J, Zachrisson BU (1982): Improving the handling properties of a composite resin for direct bonding. Am J Orthod $\underline{81}$, 269-76

Årtun J, Spadafora A, Shapiro PA (1997): A 3-year follow up study of various types of orthodontic canine-to-canine retainers. Eur J Orthod 19, 501-509

Axelsson S, Zachrisson BU (1992): Clinical experience with direct-bonded labial retainers. J Clin Orthod 29, 480-90

Bearn DR (1995): Bonded orthodontic retainers a review. Am J Orthod Dentofac Orthop $\underline{108}$, 207-13

Blechman AM (1985): Magnetic force systems in othodontics. Am J Orthod $\underline{87}$, 201-210

Bondemark L, Kurol J, Bernhold M (1994a): Repelling magnets versus superelastic nickeltitanium coils in simultaneous distal movement of first and second molars. Angle Orthod $\underline{64}$, 189-198 
Bondemark L, Kurol J, Wennberg A (1994b): Biocompatibility of new, clinically used and recycled orthodontic samarium-cobalt magnets. Am J Orthod Dentofac Orthop 105, 568-574

Bondemark L, Kurol J, Wennberg A (1994c): Orthodontic rare earth magnets- in vitro cytotoxicity assessment. Br J Orthod 21, 335-341

Bondemark L, Kurol J, Larsson A (1995): Human dental pulp and gingival tissue after static magnetic field exposure. Eur J Orthod 17, 85-91

Bryan DC, Sherrif M (1995): An in-vitro comparison between an bonded retainer system and a directly bonded flexible spiral wire retainer. Eur J Orthod 17, 143-151

Buonocore MG (1955): A simple method of increasing the adhesion of acrylic filling materials to enamel surfaces. $J$ Dent Res $\underline{34}, 849-53$

Butler J, Dowling P (2005): Orthodontic bonded retainers. J Ir Dent Assoc 51, 29-32

Carter RN (1978): Simplified direct-bonded retainer. J Clin Orthod 12, 221

Chen RS (1978): Prefabricated bonded mandibular retainer. J Clin Orthod $\underline{12}, 788-9$

Cohen S, Cassalia B, Hayes RC (1994): Direct-bonded lingual retainer with a new twist. J Clin Orthod 28(9), 538

Cook BJ (2002): A direct bonding technique for lingual retainers. J Clin Orthod $\underline{36}, 469$

Cronau M, Ihlow D, Kubein-Meesenburg D, Fanghänel J, Dathe H, Nägerl H (2006):

Biomechanical features of the periodontium:an experimental pilot study in vivo. Am J Orthod Dentofac Orthop 129(5), 599.e13-21

Dahl EH, Zachrisson BU (1991): Long-term expirience with direct-bonded lingual retainers. J Clin Orthod 25, 619-32 
Darendeliler MA, Friedle JM (1994): Treatment of an impacted canine with magnets. J Clin Orthod 28, 639-642

Darendeliler MA, Joho JP (1993): Magnetic activator device II (MADII) for correction of Class II division 1 malocclusions. Am J Orthod Dentofac Orthop 103, 223-239

Darendeliler MA, Chiarini M, Joho JP (1993): Case report: early class three treatment with magnet appliances. J Clin Orthod 27, 563-569

Dellinger EL (1986): A clinical assessment of the active vertical corrector-a nonsurgical alternative for skeletal open bite treatment. Am J Orthod $\underline{89}$, 428-436

Diamond M (1987): Resin fibreglass bonded retainer. J Clin Orthod 21, 182-3

Eade P (1980): A modified direct bond lingual retainer technique. Br J Orthod $\underline{7}, 125-6$

Federick DR (1976): A magnetically retained interim maxillary obturator. J Prosth Dent $\underline{36}$, $671-675$

Gazit E, Lieberman MA (1976): An esthetic and effective retainer for lower anterior teeth. Am J Orthod $\underline{70}, 91-3$

Gillings BR (1981): Magnetic retention for complete and partial overdentures, Part I. $J$ Prosth Dent $\underline{45}, 484-491$

Gorelick L, Geiger AM, Gwinnett AJ (1982): Incidence of white spot formation after bonding a banding. Am J Orthod $\underline{81}, 93-8$

Hahn W, Fricke J, Fricke-Zech S, Zapf A, Gruber R, Sadat-Khonsari R (2008): The use of neodymium-iron-boron magnet device for positioning a multi-stranded wire retainer in lingual retention- a pilot study in humans. Eur J Orthod $\underline{30,}$, 433-436 
Hahn W, Wasser-Merkel W, Lange K, Gruber RM, Kubein-Meesenburg D, Ihlow D (2011): Accuracy of fit of 3-to-3 retainers after adhesive fixation using a neodymium-iron-boron magnet chain. J Orofac Orthod 72(5), 381-388.

Harms V: Physik für Mediziner und Pharmazeuten. 15.Auflage, Harms Verlag, Lindhöft, 2000, 131-138

Harris LR (1990): Hard magnets. Mater Sci Technol $\underline{6}$, 962-966

Hawley CA (1919): A removable retainer. Int J Orthod $\underline{2}$, 291-8

Heier EE, De Smint AA, Wijgaerts IA, Adriaens PA (1997): Periodontal implications of bonded versus removable retainers. Am J Orthod Dentofac Orthop 112, 607-16

Hobson RS, Eastaugh DP (1993): Silicone putty splint for rapid placement of direct-bonded retainers. J Clin Orthod $\underline{27}, 536-7$

Ihlow D, Cronau M, Kubein-Meesenburg D, Heine G, Dathe H, Hansen C, Nagerl H (2003): An experimental method for in vivo analysis of biomechanical asymmetries of the periodontium. J Orofac Orthop 64(5), 321-9

Ihlow D, Cronau M, Bernitt K, Sadat-Khonsari R, Dathe H, Nägerl H, Kubein-Meesenburg D (2005): The retention catalogue: an instrument for quality management. J Orofac Orthop $\underline{66}$, $377-87$

Javid N (1971): The use of magnets in a maxillofacial prothesis. J Prosth Dent $\underline{25}$, 334-341

Kahl-Nieke B: Retention, Stabilität, Rezidiv. In: Dietrich P (Hg.) Praxis der Zahnheilkunde. Bd. Kieferorthopädie III. 4. Aufl. Urban \& Fischer, München-Jena 2002, 209-39

Kaplan H (1988): The logic of modern retention procedures. Am J Orthod Dentofac Orthop $\underline{93}, 325-40$

Knierim RW (1973): Invisible lower cuspid to cuspid retainer. Angle Orthod $\underline{43}$, 218-9 
Lee KD, Mills CM (2009): Bond failure rates for V-loop vs straight wire lingual retainers. Am J Orthod Dentofac Orthop 135, 502-6

Lee RT (1981): The lower incisor bonded retainer in clinical practice: a three year study. $B r J$ Orthod $\underline{8}, 15-8$

Li H (2007): Magnet decoration, beautiful but potentially dangerous for patients with implantable pacemakers or defibrillators. Heart Rhythm $\underline{4}, 5-6$

Little RM (1975): The irregularity Index: a quantitative score of mandibular anterior alignment. Am J Orthod $\underline{68}, 554-563$

Little RM (1990): Stability and relapse of dental arch alignment. Br J Orthod 17, 235-241

Little RM, Wallen TR, Riedel RA (1981): Stability amd relapse of mandibular anterior alignment-first premolar extraction cases treated by traditional edgewise orthodontics. Am J Orthod 80(4), 349-365

Little RM, Riedel RA, Artun J (1988): An evaluation of changes in mandibular anterior

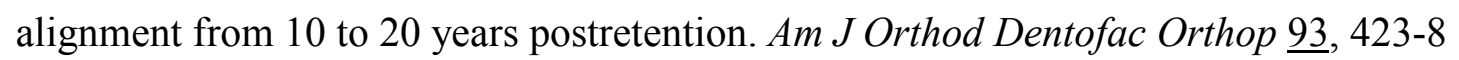

Lubit EC (1979): The bonded lingual retainer. J Clin Orthod $\underline{13}, 311-3$

Lumsden K, Saidler G, Mc Coll JH (1999): Breakage Incidence with direct bonded lingual retainers. Br J Orthod 26, 191-194

Melrose C, Millett DT (1998): Toward a perspective on orthodontic retention? Am J Orthod Dentofac Orthop 113(5), 507-14

Meyers CE, Vogel S (1982): Stabilization of retainer wire for direct bonding. J Clin Orthod $\underline{16}, 412$

Moser U, Moser L (1996): Langzeitretention mit geklebten lingualen Glasfaserertainern. Kieferorthopädie 10, 85-94 
Moyers, RE: Handbook of orthodontics for the student and general practitioner. $3^{\text {rd }} \mathrm{ed}$, Yearbook; Chicago 1973, 442

Nanda RS, Nanda SK (1992): Considerations of dentofacial growth in long-term retention and stability: is active retention needed? Am J Orthod Dentofac Orthop 101, 297-303

Newman GV (1965): Epoxy adhesives for orthodontic attachments: progress report. Am J Orthod $\underline{51}, 901-12$

Noar JH, Evans RD (1999): Rare earth magnets in orthodontics: an overview. Br J Orthod $\underline{26}, 29-37$

Orchin JD (1990): Permanent lingual bonded retainer. J Clin Orthod 24, 229-31

Osborn DB (1983): Bonded lingual retainers. Am J Orthod $\underline{83}, 218$

Paulson RC (1992): A functional rationale for routine maxillary bonded retention. Angle Orthod $\underline{62}, 223-6$

Radlanski RJ, Zain N (2004): Stability of bonded lingual wire retainer-a study of the initial bond strength. J Orofac Orthop $\underline{65}, 321-35$

Read MJF (1984): The bonding of orthodontic attachments using a visible light cured adhesive. Br J Orthod $11,16-20$

Reitan K (1967): Clinical and histologic observations on tooth movement during and after orthodontic treatment. Am J Orthod 53(10), 721-45

Reitan K (1969): Principles of the retention and avoidance of posttreatment relapse. Am J Orthod 55, 779-90

Riedel RA (1960): A review of the retention problem. Angle Orthod $\underline{30}$, 179-94 
Rose E, Frucht S, Jonas IE (2002): Clinical comparison of a multistranded wire and a directbonded polyethylene ribbon-reinforced resin composite used for lingual retention. Quintessence Int $\underline{33(8)}$, 579-83

Rosenberg S (1980): A new method of stabilization of periodontally involved teeth. J Periodontol $\underline{51}, 469-73$

Rubenstein BM (1976): A direct bond maxillary retainer. J Clin Orthod 10, 43

Sandler JP (1991): An attractive solution to unerupted teeth. Am J Orthod Dentofac Orthop $\underline{100}, 489-493$

Sandler JP, Meghi S, Murray AM, Springate SD, Sandy JR, Crow V, Reed RT (1989):

Magnets in Orthodontics. Br J Orthod 16, 139-141

Schütz-Fransson U, Bjerklin K, Kurol J (1998): Long-term development in the mandible and incisor crowding with and without an orthodontic stabilising appliance. J Orofac Orthop $\underline{59}$, $63-72$

Schwarze J, Bourauel C, Drescher D (1995): Frontzahnbeweglichkeit nach direkter Klebung von Lingualretainern. Fortschr Kieferorthop 56, 25-33

Segner D, Heinrici B (2000): Bonded retainers-Clinical reliability. J Orofac Orthop $\underline{5}$, 352358

Shah AA, Sandler PJ, Murray AM (2005): How to...place a lower bonded retainer. J Orthod $\underline{32}, 206-210$

Springate SD, Sandler PJ (1991): Micro-magnetic retainers: an attractive solution to fixed retention. Br J Orthod $\underline{18}, 139-141$

Störmann I, Ehmer U (2002): A Prospective randomized study of different retainer types. J Orofac Orthop $\underline{63}, 42-50$ 
Vardimon AD (1987): Magnetic versus mechanical expansion with different force threholds and points of application. Am J Orthod Dentofac Orthop $\underline{92}$, 455-466

Vardimon AD, Mueller HJ (1985): In vivo and in vitro corrosion of permanent magnets in orthodontic therapy. $J$ Dent Res $\underline{64}, 89$

Vardimon AD, Stutzmann JJ, Graber TM, Voss LR, Petrovic AG (1989): Functional orthopaedic magnetic appliance (FOMA) II-Modus operandi. Am J Orthod Dentofac Orthop $\underline{95}, 371-387$

Vardimon AD, Graber RM, Voss LR, Muller TP (1990): Functional orthopaedic appliance (FOMA) III-Modus operandi. Am J Orthod Dentofac Orthop 97, 135-148

Watted N, Wieber M, Teuscher T, Schmitz N (2001): Comparison of incisor mobility after insertion of canine-to-canine lingual retainers bonded to two or to six teeth. J Orofac Orthop $\underline{62(5)}, 387-396$

Weisser JI (1978): Bondable twin lower 3-3 teaining arch. J Clin Orthod 12, 557-60

Wilson M, Kpendema M, Noar JH, Hunt NP, Mordan NJ (1995): Corrosion of intra-oral magnets by biofilms of Streptococcus sanguis. Biomaterials $\underline{16}, 721-725$

Wilson M, Patel H, Kpendema M, Noar JH, Hunt NP, Mordan NJ (1997): Corrosion of intraoral magnets by multi-species biofilms in the presence and absence of sucrose. Biomaterials $\underline{18}, 53-57$

Wolber T, Ryf S, Binggeli C, Holzmeister J, Brunckhorst C, Luechinger R, Duru F (2007):

Potential interferences of small neodymium magnets with cardiac pacemakers and implantable cardioverter-defibrilators. Heart Rhythm $\underline{4}, 1-4$

Zachrisson BU (1977): Clinical experience with direct-bonded orthodontic retainers. Am J Orthod 71, 440-8 
Zachrisson BU (1982): The bonded lingual retainer and multiple spacing of anterior teeth. Swed Dent J $\underline{15}, 247-255$

Zachrisson BU (1983): The bonded lingual retainer and multiple spacing of anterior teeth. J Clin Orthod 17, 838-44

Zachrisson BU: Bonding in Orthodontics. Orthodontics: current principles and techniques, C.V. Mosby Company, St-Louis 1985, 440-8

Zachrisson BU (1986): Excellence in finishing. J Clin Orthod 20, 460-82 und 536-56

Zachrisson BU (1995): Third-Generation mandibular bonded lingual 3-3 retainer. J Clin Orthod 29(1), 39-48

Zachrisson BU (1997): Important aspects of long-term stability. J Clin Orthod 31, 562-583

Zekic E, Gelgör IE (2004): An acrylic transfer tray for direct-bonded lingual retainers. J Clin Orthod $\underline{38(10)}, 551-553$ 
$8 \quad$ Anhang

8.1 Arbeitsanweisung für Zeitmess- und Passgenauigkeitsstudie 3-3Retainer

Arbeitsanweisung für Zeitmess- und Passgenauigkeitsstudie 3-3Retainer

Modellnummer:

Gruppe:

1. Kleben eines 0,015Twistflex-Retainers ( 6-Basen ) mit konventioneller Positionierhilfe

Zeit [ $\mathrm{min}][\mathrm{sec}]$ :

Abstandsfläche Draht/Zähne [Pixel]

Messung 1:

Messung 2:

2. Kleben eines 0,018 Twistflex-Retainers ( 6-Basen ) mit konventioneller Positionierhilfe

Zeit [ $\mathrm{min}][\mathrm{sec}]$ :

Abstandsfläche Draht/Zähne [Pixel]

Messung 1:

Messung 2:

3.a) Aufbringen der Magnetkette

Zeit [ $\min ][\mathrm{sec}]$ :

3.b) Kleben eines 0,015 Twistflex-Retainers ( 6-Basen ) mit magnetischer Positionierhilfe Zeit [ $\mathrm{min}][\mathrm{sec}]$ : Abstandsfläche Draht/Zähne [Pixel] Messung 1: Messung 2:

3.c) Kleben eines 0,018 Teistflex-Retainers ( 6-Basen ) mit magnetischer Positionierhilfe 
Zeit [ $\mathrm{min}][\mathrm{sec}]$ :

Abstandsfläche Draht/Zähne [Pixel]

Messung 1:

Messung 2:

3.d) Entfernen der Magnetkette

Zeit [ $\min ][\mathrm{sec}]$ :

$\mathrm{Zu} \mathrm{1.,} \mathrm{2.:}$

Die Zeiterfassung beginnt mit dem Aufnehmen des Retainers vom Tray. Anschließend wird der Retainer auf das Modell aufgesetzt, in der Horizontalebene ausgerichtet und seine Passgenauigkeit kontrolliert. Daraufhin wird der Retainer wieder abgenommen und das Adhäsiv wird aufgetragen, welches 2 x $30 \mathrm{sec}$. unter einer UV-Lampe ausgehärtet werden muss. Der Retainer wird erneut aufgesetzt und der Kunststoff Teric flow von Heraeus Kulzer wird aufgetragen. Dieser wird ebenfalls 2 x $30 \mathrm{sec}$. ausgehärtet. Mit dem Entfernen der Einbringhilfe endet die Zeitmessung.

$\mathrm{Zu} \mathrm{3.a):}$

Die Magnetkette liegt auf einer Wachsstange aufgepresst auf dem Tray. Die Zeitmessung beginnt mit der Aufnahme der Magnetkette vom Tray und endet nach dem Andrücken der Magnetkette.

Zu 3.b) und 3.c):

Die Zeiterfassung beginnt mit dem Aufnehmen des Retainers vom Tray. Anschließend wird der Retainer auf das Modell aufgesetzt, in der Horizontalebene ausgerichtet und seine Passgenauigkeit kontrolliert. Daraufhin wird der Retainer wieder abgenommen und das Adhäsiv wird aufgetragen, welches 2 x $30 \mathrm{sec}$. unter einer UV-Lampe ausgehärtet werden muss. Der Retainer wird erneut aufgesetzt und der Kunststoff Transbond LR (3M Unitek, Monrovia, California, USA) wird aufgetragen. Die Zeitmessung endet nach dem Aushärten des Kunststoffes, welches 2 x 30 sec. Zeit benötigt.

$\mathrm{Zu} \mathrm{3.d):}$

Die Zeiterfassung startet mit der Entfernung der Magnetkette sowie des Wachses von den lingualen Zahnoberflächen und endet, wenn beides vollständig entfernt wurde. 


\subsection{Arbeitsanweisung für die Herstellung der 3-3-Retainer durch den Zahntechniker}

\section{Arbeitsanweisung für die Herstellung der 3-3-Retainer durch den Zahntechniker}

1. Herstellung eines 3-3 Retainers aus 0.015 Twistflex Draht mit Positionierhilfe

Modellnummer und Gruppe:

Zeit [min] [sec]:

2. Herstellung eines 3-3 Retainers aus 0.018 Twistflex Draht mit Positionierhilfe

Modellnummer und Gruppe:

Zeit [min] [sec]:

3. Herstellung eines 3-3 Retainers aus 0.015 Twistflex Draht mit Magnetkette

Modellnummer und Gruppe:

Zeit [min] [sec]:

4. Herstellung eines 3-3 Retainers aus 0.018 Twistflex Draht mit Magnetkette

Modellnummer und Gruppe:

Zeit [min] [sec]:

$\mathrm{Zu} \mathrm{1.,2.:}$

Die Zeitmessung beginnt mit dem Isolieren der vorliegenden Gipsmodelle mit Isolant (Dentsply), einem Isolationsmittel für Gips/Kunststoff. Die Überschüsse des Isolationsmittels werden entfernt und der entsprechende Draht (0,015 Dentaflex, 0,018 Dentaflex von Dentaurum) wird gebogen. Der fertig gebogene Retainer wird mit Wachs an den Eckzähnen des Gipsmodells befestigt und die Positionierungshilfe wird mit Vita VM LC lichthärtendem Kunststoff hergestellt. Anschließend wird der Kunststoff für $30 \mathrm{sec}$. unter einer UV-Lampe ausgehärtet. Anschließend wird das Wachs mit Hilfe eines Dampfstrahlgerätes entfernt und 
der Retainer mit der Positionierungshilfe vom Modell entfernt. Abschließend erfolgt eine Kontrolle der Positionierungshilfe und eventuelle Korrekturen. Die Zeitmessung endet an dem Zeitpunkt, an dem der Techniker den Retainer mit Einsatzhilfe für einsatzfähig erklärt.

\section{$\mathrm{Zu} 3 ., 4 .:$}

Die Zeitmessung startet mit der Befestigung der Magnetkette an den vestibulären Oberflächen der Frontzähne mit Hilfe von Wachs. Anschließend wird der Retainer aus dem entsprechenden Draht (0,015 Dentaflex, 0,018 Dentaflex von Dentaurum) gebogen. Mit der Entfernung der Magnetkette und dem Wachs vom Gipsmodell endet die Zeitmessung. 


\subsection{Arbeitsanweisung für das Ausmessen der Bilddateien}

\section{Arbeitsanweisung für das Ausmessen der Bilddateien}

Bei den mit 3-3-Retainern versehenen Modellen wird das Segment zwischen Zahn 33 - 43 herausgetrennt. Danach erfolgt die Reduzierung der mit dem Retainer versehenen Gipszähne in der Vertikalen parallel bis auf Höhe des Retainerdrahtes an einem Feintrimmer. Die so gewonnenen Segmente werden mit Hilfe eines Flachscanners (Epson Perfection 750V pro, Epson Deutschland $\mathrm{GmbH}$, Meerbusch, Deutschland) in der Auflösung $3200 \times 3200$ Pixel/Zoll eingescannt. Die so erstellten Bilddateien werden in das Histomorphometrie-Programms KS 300 (Carl Zeiss, Jena, Deutschland) übertragen.

Mit Hilfe des Programms erfolgt die Einzeichnung eines Feldes (Fläche $\left[\mathrm{mm}^{2}\right]$ ) zwischen der den Labialflächen der Zähne zugewandten Oberfläche des Drahtes und den labialen Begrenzungen der beklebten Zähne. Die Linienbegrenzung des Messfeldes läuft dabei tangential entlang der beschriebenen Grenzen. Um Längenunterschiede der Drähte bei der Flächenmessung zu kompensieren, wurde der jeweils gemessene Endpunkt des Drahtes folgendermaßen festgelegt:

- Die maximale mesio-distale Ausdehnung der endständigen Eckzähne wird auf Höhe des Drahtes bestimmt. Dieser Wert wird gedrittelt. Der Endpunkt des Drahtes liegt bei den Messungen nach zwei Dritteln der mesio-distalen Ausdehnung der endständigen Eckzähne.

- Von jedem Zahn wird die Distanz [mm] zwischen der oralen Kante eines jeden beklebten Zahnes in der Mitte seiner mesio-distalen Ausdehnung senkrecht zu einer Tangente der nach labial gerichteten Kante des Retainerdrahtes eines jeden beklebten Zahnes gemessen.

- $\quad$ Alle Messungen werden durch einen Untersucher durchgeführt. Jedes Bild wird zweimal vermessen.

Alle Messwerte werden mit Hilfe des Programms Excel 2007 (Microsoft, Redmon, WA, USA) tabellarisch erfasst. 


\section{Danksagung}

Meinem Doktorvater PD. Dr. W. Hahn möchte ich für die freundliche Überlassung des Themas sowie die engagierte Förderung dieser Dissertation herzlich danken. Ohne seine außerordentlich gute Betreuung und die fortwährend hilfsbereite Unterstützung wäre diese Arbeit nicht möglich gewesen.

Danken möchte ich ebenfalls Herrn Jens Köhler sowie Herrn Sven Köhler für die großartige Betreuung der experimentellen Arbeiten.

Ein weiterer Dank gilt der Abteilung für Mund-, Kiefer-, Gesichtschirurgie unter Leitung von Herrn Prof. Dr. Dr. H. Schliephake für die freundliche Bereitstellung ihrer Laborräumlichkeiten zur Vermessung der untersuchten Modelle. Ein besonderer Dank gilt Herrn PD. Dr. Dr. R. Gruber für die Einweisung in das automatische Bildanalysesystem KS 300.

Abschließend bedanke ich mich bei Prof. Dr. T. Friede und Frau Dr. K. Lange für ihre aktive Unterstützung bei der statistischen Auswertung der vorliegenden Ergebnisse. 


\section{Lebenslauf}

Am 4. Oktober 1983 wurde ich als zweites Kind von Dr. med. Jörg Martin Merkel und seiner Ehefrau Gabriele Dorothea Anna Osterloh-Merkel, geb. Osterloh, in Marburg geboren. Ich bin verheiratet und habe eine Tochter.

1989 erfolgte meine Einschulung in die Grundschule in Großseelheim. Ab 1990 besuchte ich die Grundschule in Alheim-Heinebach. Im Anschluss besuchte ich von 1993 bis 1995 die Förderstufe in Rotenburg/Fulda. 1995 wechselte ich dann auf die Jakob-Grimm-Schule Rotenburg/Fulda. Die Allgemeine Hochschulreife erlangte ich 2002.

Zum Wintersemester 2002/2003 nahm ich das Studium der Zahnheilkunde an der GeorgAugust-Universität Göttingen auf. Im September 2003 legte ich die Naturwissenschaftliche Vorprüfung ab, im März 2005 die Zahnärztliche Vorprüfung und die Zahnärztlich Prüfung im November 2007. Die Approbation wurde mir im Dezember 2007 erteilt.

Von Februar 2008 bis Oktober 2008 war ich als Assistenzzahnärztin in der Zahnarztpraxis Dr. Gäbert-Gallo in Kassel beschäftigt. Anschließend war ich von Oktober 2008 bis März 2010 als Assistenzzahnärztin und von März 2010 bis Juli 2012 als angestellte Zahnärztin in der Zahnarztpraxis Dres. Plewe und Hilgenberg in Kassel tätig. Seit Juli 2012 bin ich in der Gemeinschaftspraxis Dr. med. dent. M. Wasser / W. Wasser-Merkel in Bad Emstal niedergelassen.

Im Juli 2006 begann ich in der Arbeitsgruppe von PD Dr. W. Hahn mit den Versuchen zur vorliegenden Dissertation. Die Ergebnisse der Dissertation wurden bereits international publiziert: Hahn W, Wasser-Merkel W, Lange K, Gruber R, Kubein-Meesenburg D, Ihlow D (2011): Accuracy of fit of 3-to-3 retainers after adhesive fixation using a neodymium - iron boron magnet chain . J Orofac Ortop 72 (5), 381-388 